\title{
Interventions to increase personal protective behaviours to limit the spread of respiratory viruses: A rapid evidence review and meta-analysis
}

\author{
Olga Perski ${ }^{1}$, Dorothy Szinay ${ }^{2}$, Elizabeth Corker ${ }^{1}$, Lion Shahab $^{1}$, Robert West ${ }^{1}$, Susan Michie $^{1}$ \\ 1 University College London, University of London \\ 2 University of East Anglia
}

Funding: The author(s) received no specific funding for this work.

Potential competing interests: The author(s) declared that no potential competing interests exist.

\section{Abstract}

Purpose: Changing human behaviour is critical for stopping the spread of respiratory viruses, including SARS-CoV-2. This includes increasing personal protective behaviours: we need evidence to inform how to achieve this. We aimed to evaluate the acceptability, practicability, effectiveness, affordability, spill-over effects and equity impact of interventions to increase personal protective behaviours to limit the spread of respiratory viruses.

Methods: We used standard best practice for rapid evidence reviews. We searched Ovid MEDLINE and Scopus to identify interventions designed to change six personal protective behaviours: hand hygiene; avoiding touching the 'TZone'; catching droplets in tissues; face mask use; disinfecting surfaces; and maintaining physical distancing. Primary research studies conducted in adults or children with active or passive comparators were included. A narrative synthesis and random-effects meta-analyses were conducted.

Results: We identified 39 studies conducted across 15 countries. Interventions targeted hand hygiene $(n=30)$ and/or face mask use $(n=12)$ and used two- or three-arm study designs with passive comparators. Interventions were typically delivered face-to-face and included a median of three behaviour change techniques. Interventions to increase hand hygiene had a medium, positive effect $\left(d=0.62,95 \% \mathrm{Cl}=0.43-0.80, p<.001, \mathrm{I}^{2}=81.2 \%\right)$. Interventions targeting face mask use had mixed results, with an imprecise pooled estimate $\left(\mathrm{OR}=4.14,95 \% \mathrm{Cl}=1.24-13.79, p<.001, \mathrm{I}^{2}=89.67 \%\right)$. Between-study heterogeneity was high.

Conclusions: We found positive effects of interventions targeting hand hygiene, with unclear results for interventions targeting face mask use.

Introduction 
Respiratory viruses such as SARS-CoV-2 enter the body through the eyes, nose and mouth (the 'T-Zone') ${ }^{1}$. Changing human behaviour is critical for stopping the spread of the SARS-CoV-2 virus and for supporting the easing of financially and psychologically costly physical distancing measures ${ }^{2-5}$. Personal protective behaviours, including hand washing, disinfecting fomites such as clothes or furniture, and face mask wearing, are advocated for limiting the spread of SARSCoV-2 $2^{6,7}$. Simply advising people to adopt these behaviours has been found to be insufficient, just as has explaining what to do and why these behaviours are necessary ${ }^{8}$. Directly relevant evidence on interventions to promote adherence to personal protective behaviours in community-dwelling children and adults is sparse but there is an urgent need to identify and synthesise what evidence does exist. Policymakers need evidence to inform the development of public health guidance and decide which interventions to prioritise. We adopted best practice for rapid evidence reviews to evaluate the acceptability, practicability, effectiveness, affordability, spill-over effects (unintended consequences) and equity impact (the 'APEASE' criteria ${ }^{9}$ ) of interventions to increase personal protective behaviours.

During pandemics of respiratory viruses, multipronged approaches involving both pharmacological (e.g. vaccination) and behavioural measures (e.g. hand washing, physical distancing) are required to bring the reproductive number below $1^{2-}$ 4,10 . Vaccination of populations will take months, even years, to roll out, especially in low- and middle-income countries. Hence, physical distancing and other behavioural measures will be required, possibly permanently. Population-wide restrictions are costly from financial, social and psychological perspectives: the world economy has been projected to shrink by approximately $4.9 \%$ in $2020^{11}$, with an additional 88 million people globally being pushed into extreme poverty (i.e. living on less than $\$ 1.90 / \mathrm{day})^{12}$, and prolonged periods of social isolation are associated with increases in domestic violence ${ }^{13}$ and negative mental health effects, such as post-traumatic stress disorder, confusion and anger ${ }^{14}$. Less costly, yet highly effective ${ }^{15}$, personal protective behaviours are thus important for supporting the easing of lockdown measures to ensure long-term suppression of viral transmission and preparedness for new viral waves and future pandemics ${ }^{3,5,10}$. To successfully block transmission, personal protective behaviours must be adopted across the population. Although systematic reviews of interventions to change hand hygiene in healthcare professionals are available ${ }^{16-20}$, generalisability to community settings is limited. There also appears to be little evidence about interventions to change behaviours such as not touching the T-Zone (eyes, nose and mouth), which would have a significant effect if adopted ${ }^{21}$ and carry little or no costs to people or society. If adopted at scale across the population including disadvantaged communities, such interventions have the potential to reduce health inequalities. Here, we aimed to conduct a rapid evidence review to evaluate the acceptability, practicability, effectiveness, affordability, spill-over effects and equity of interventions to increase personal protective behaviours that limit the spread of respiratory viruses.

\section{Methods}

\section{Study design}

The study protocol was pre-registered on the Open Science Framework (https://osf.io/7cphy). During ongoing pandemics, the World Health Organization recommends the use of rapid evidence reviews for swift knowledge generation ${ }^{22}$. We 
adopted acknowledged best practice for rapid evidence reviews, which involved completing the review in a timely fashion, limiting the search to main databases and the published literature, and having one reviewer extract data and another verify 23,24 .

\section{Criteria for considering studies for this review}

\section{$\underline{\text { Population }}$}

We included studies that recruited as participants community-dwelling children or adults (as opposed to qualified or trainee healthcare professionals in hospital or care home settings).

\section{Intervention}

We included reports of evaluations of interventions designed to increase at least one of six personal protective behaviours to block transmission of respiratory viruses (see Figure 1, coloured rectangles).

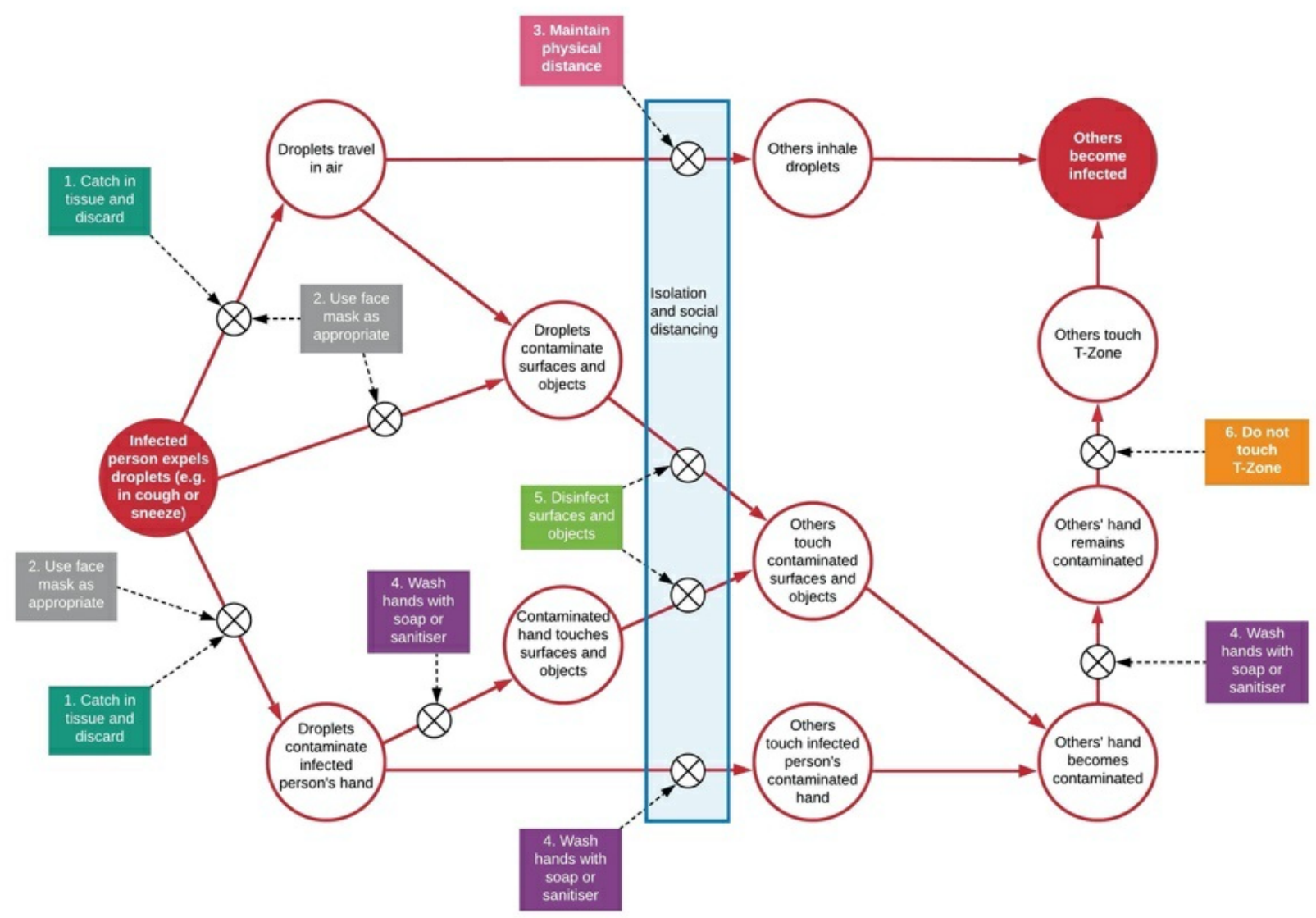

Figure 1. Map of personal protective behaviours relevant for blocking transmission of SARS-CoV-2: hand washing and use of hand sanitizers; avoiding touching the 'T-Zone'; catching droplets in tissues and discarding these; face mask use; disinfecting surfaces; and maintaining physical distancing. Reproduced with permission from the authors ${ }^{25}$. 


\section{Comparison}

We included studies with an active or passive (e.g. wait-list control, baseline) comparator.

\section{Outcomes}

We included studies that reported as outcome either the acceptability, practicability, effectiveness, affordability, spill-over effects and/or equity of interventions, measured via self-report or direct observation.

\section{Study designs}

We included primary research studies that used experimental (e.g. randomised controlled trial) or quasi-experimental (e.g. pre- and post-test) study designs, providing that they were conducted under free-living (as opposed to laboratory) conditions. In line with rapid review guidelines, we only included studies that were published in peer-reviewed journals and written in English ${ }^{22,23}$.

\section{Search methods for identification of studies}

\section{Electronic searches}

We searched Ovid MEDLINE and Scopus. Search terms for each behaviour (e.g. 'hand hygiene', 'hand washing', 'face mask') were piloted and refined to achieve balance between sensitivity and specificity (see Supplementary file 1).

\section{Searching for other sources}

Expertise within the review team and consultation with topic experts was used to identify additional articles of interest. We had specified the use of reference chaining in the review protocol; however, given the rapid focus of the review and large number of identified studies, reference chaining was not performed.

\section{Data collection and analysis}

\section{Selection of studies}

Two reviewers (OP, DS) independently screened i) titles and abstracts and ii) full texts against the inclusion criteria.

Discrepancies were discussed and resolved through consulting with a third reviewer (EC) if necessary. 


\section{Data extraction and management}

A data extraction form was developed on the basis of the Behaviour Change Intervention Ontology (www.humanbehaviourchange.org) and Cochrane's PICO ontology (https://linkeddata.cochrane.org/pico-ontology). Ontologies are classification systems which enable researchers to specify entities and their inter-relationships, thus facilitating systematic knowledge synthesis ${ }^{26}$. We extracted data on study design, intervention content (i.e. behaviour change techniques (BCTs), coded against the BCT Taxonomy $\mathrm{v} 1{ }^{27}$ ), mode of delivery, population, setting, and mechanism(s) of action ${ }^{28,29}$. As an intervention may be effective but have negative spill-over effects to other behaviours, or be impracticable and/or unacceptable to key stakeholders, we extracted data pertaining to the APEASE criteria (see Table $1)^{9}$. Data were extracted by one reviewer (OP or DS). In the review protocol, we had specified that extracted data would be verified by a second reviewer to assess accuracy and completeness. However, given the large number of identified studies, a second reviewer (EC) verified $10 \%$ of studies.

Table 1. APEASE criteria for evaluating intervention approaches or components ${ }^{9}$.

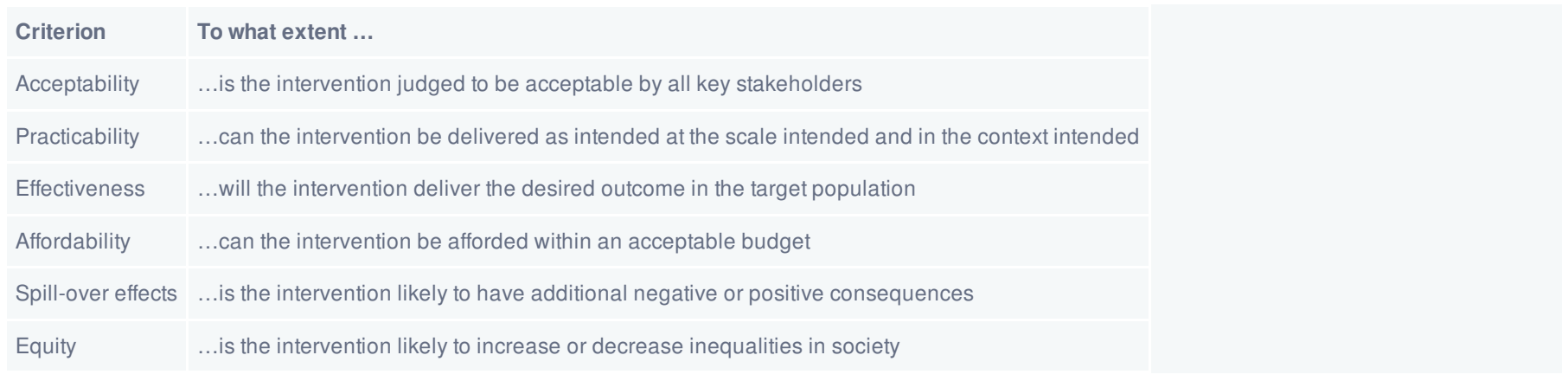

\section{Quality appraisal}

The methodological rigour of included evaluation reports was assessed by one reviewer (OP or DS) using Cochrane's risk of bias tool ${ }^{30}$. A second reviewer (EC) verified $10 \%$ of studies.

\section{Stakeholder involvement}

We solicited input from key stakeholders, including patient and public representatives recruited via panels convened by Public Health England $(n=282)$ and the University of East Anglia $(n=3)$, and UK policymakers and academic researchers contacted via a mailing list on the research objectives, target behaviours and outcomes assessed. Feedback from patient and public representatives $(n=20)$ was incorporated into the review protocol; we did not receive any suggestions for improvement or clarification from the policymakers and academic researchers. The rapid review results will be disseminated to stakeholders via an infographic.

\section{Data synthesis}


A narrative (descriptive) synthesis was conducted for each of the six personal protective behaviours. We had specified in the review protocol that meta-analyses would be conducted if practicable and appropriate (i.e. $>5$ studies with homogeneous study designs and outcome variables). After inspection of study designs and outcome variables, however, we deemed it useful to conduct a meta-analysis with $\mathrm{k}=4$ studies. Random-effects meta-analyses to estimate a pooled odds ratio (OR) or the standardised mean difference (d) were conducted in RStudio v.1.2.5033 with the metafor package ${ }^{31}$. Cohen's conventions for small $(d=0.2)$, medium $(d=0.5)$ and large $(d=0.8)$ effects were used in the interpretation of the results ${ }^{32}$. Where studies reported more than one hand hygiene outcome (e.g. hand washing and hand sanitizer use), only the first reported outcome was included in the meta-analysis, so as not to violate the assumption of independence ${ }^{33}$. Where studies did not report sufficient detail to calculate effect sizes, study authors' own description/interpretation of results were grouped into 'positive' effects (i.e. a significant difference between intervention and control groups, favouring the intervention group, was detected), 'no difference' (i.e. a significant difference between groups was not detected), 'negative' effects (i.e. a significant difference between groups, favouring the control group, was detected) or 'indeterminate' (i.e. differences between groups were not reported or could not be computed given the study design). To aid interpretation, for behaviours where a majority of positive or negative results were observed, overall results were categorised as either 'positive' or 'negative', respectively, and were categorised as 'mixed' if a clear majority was not observed or where the majority of results were categorised as 'indeterminate'.

\section{Results}

\section{Study selection}

After removing duplicates, 5,594 records were identified, with 158 studies carried forward to the full text screening. Of the 39 studies included in the narrative evidence synthesis, 10 were included in meta-analyses (see Figure 2). 


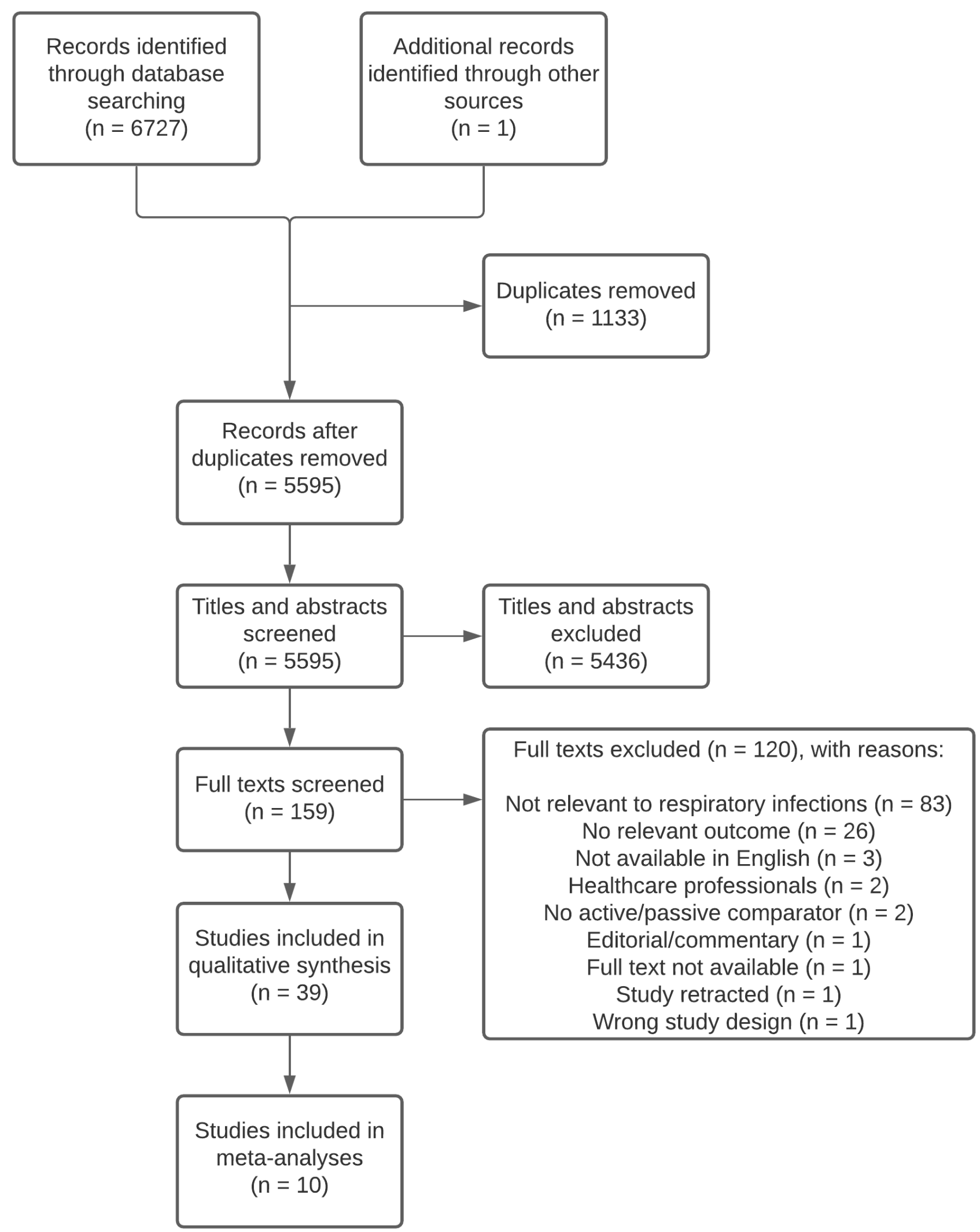

Figure 2. PRISMA flow chart of included studies. 
Studies were conducted in the United States $(13 / 39 ; 33 \%)^{34-46}$, China $(5 / 39 ; 13 \%)^{47-51}$, Germany $(3 / 39 ; 13 \%)^{52-54}$, Thailand $(3 / 39 ; 13 \%)^{55-57}$, Australia $(2 / 39 ; 5 \%)^{58,59}$, Denmark $(2 / 39 ; 5 \%)^{60,61}$, Spain $(2 / 39 ; 5 \%)^{62,63}$, the United Kingdom $(2 / 39 ; 5 \%)^{64,65}$, with one study each in Australia/Saudi Arabia ${ }^{66}$, Bangladesh ${ }^{67}$, Costa Rica ${ }^{68}$, Finland ${ }^{69}$, France ${ }^{70}$, the Netherlands ${ }^{71}$, and Turkey ${ }^{72}$ (see Table 2). Studies had a median of 419 participants (range: 96 to 20,066).

The majority of studies $(28 / 39 ; 72 \%)$ targeted hand hygiene, with the remaining studies targeting a combination of hand hygiene and/or face mask use $(9 / 39 ; 23 \%)^{37,38,43,48,49,52,53,55,59}$, face mask use only $(3 / 39 ; 8 \%)^{61,66,70}$ or a combination of catching of droplets in tissues and hand hygiene $(1 / 39 ; 3 \%)^{50}$ (see Table 2). Interventions were delivered in participants' own homes (12/39; $31 \%)$, nurseries/schools (10/39; $26 \%)$, university residence halls/public areas $(5 / 39 ; 13 \%)$, offices $(4 / 39 ; 10 \%)$, online $(2 / 39 ; 5 \%)$, outpatient clinics $(1 / 39 ; 3 \%)$, or an army training centre $(1 / 39 ; 3 \%)$. Four studies did not state the setting for intervention delivery. Studies targeted children and adult household members (16/39; $41 \%)$, pre- or school children and/or teachers $(8 / 39 ; 21 \%)$, university staff and/or students $(5 / 39 ; 13 \%)$, office workers $(4 / 39 ; 10 \%)$, communitydwelling adults $(3 / 39 ; 8 \%)$, Hajj pilgrims $(1 / 39 ; 3 \%)$, military trainees $(1 / 39 ; 3 \%)$ or adult members of a social service centre $(1 / 39 ; 3 \%)$.

Study designs used were two-arm, cluster RCTs (11/39; 28\%), three-arm, cluster RCTs (10/39;26\%), two-arm RCTs (5/39; $13 \%)$, single-arm, pre- and post-intervention studies (5/39; 13\%), two-arm, non-randomised cohort studies (2/39; 5\%), three-arm RCTs (2/39; 5\%), two-arm, pilot RCTs (1/39; 3\%) or randomised, cross-over studies (1/30; 3\%).

\section{Intervention characteristics}

Intervention durations ranged from one day (i.e. one-off interventions) to three years (see Table 3). In studies using a twoor three-arm design (33/39; 85\%), comparators included no intervention/usual care (19/33; 58\%), educational materials $(9 / 33 ; 27 \%)$, the provision of soap/hand sanitizer $(3 / 33 ; 9 \%)$ or a combination of educational materials and soap/hand sanitizer (2/33; 6\%). Interventions were delivered via face-to-face sessions (23/39; 59\%), written materials (including books and newsletters) (14/39; $36 \%)$, posters/bulletin boards/cue cards (8/39; $21 \%)$, cartoons/games (4/39; 10\%), videos (4/39; $10 \%)$, telephone $(3 / 39 ; 8 \%)$ and/or websites $(2 / 39 ; 5 \%)$. Five studies did not clearly report on the mode of intervention delivery. Where reported, participants received a flat payment for study completion $34,37,49,52,53,55,73$, payment per survey completed $^{40}$, points for study completion ${ }^{36}$ or points per survey completed ${ }^{39}$.

We coded 15 different BCTs across interventions (see Figure 3); however, details on intervention content were typically lacking. Interventions included a median of three BCTs (range: 0 to 6). The most frequently coded BCTs were '12.5 Adding objects to the environment' (17/39; 44\%), ' 4.1 Instruction on how to perform the behaviour' (16/39; 41\%) and '5.1 Information about health consequences' (10/39; 26\%). Few studies reported targeting a specific mechanism of action. Where reported, interventions were designed to target intentions ${ }^{64}$, attitudes ${ }^{35,64}$, subjective norms ${ }^{35,64}$, perceived behavioural control ${ }^{47,64}$, perceived risk of infection/disease severity ${ }^{35,47,64}$, action control ${ }^{68}$, coping planning ${ }^{68}$, 
knowledge ${ }^{47,51}$ or skills ${ }^{51}$.

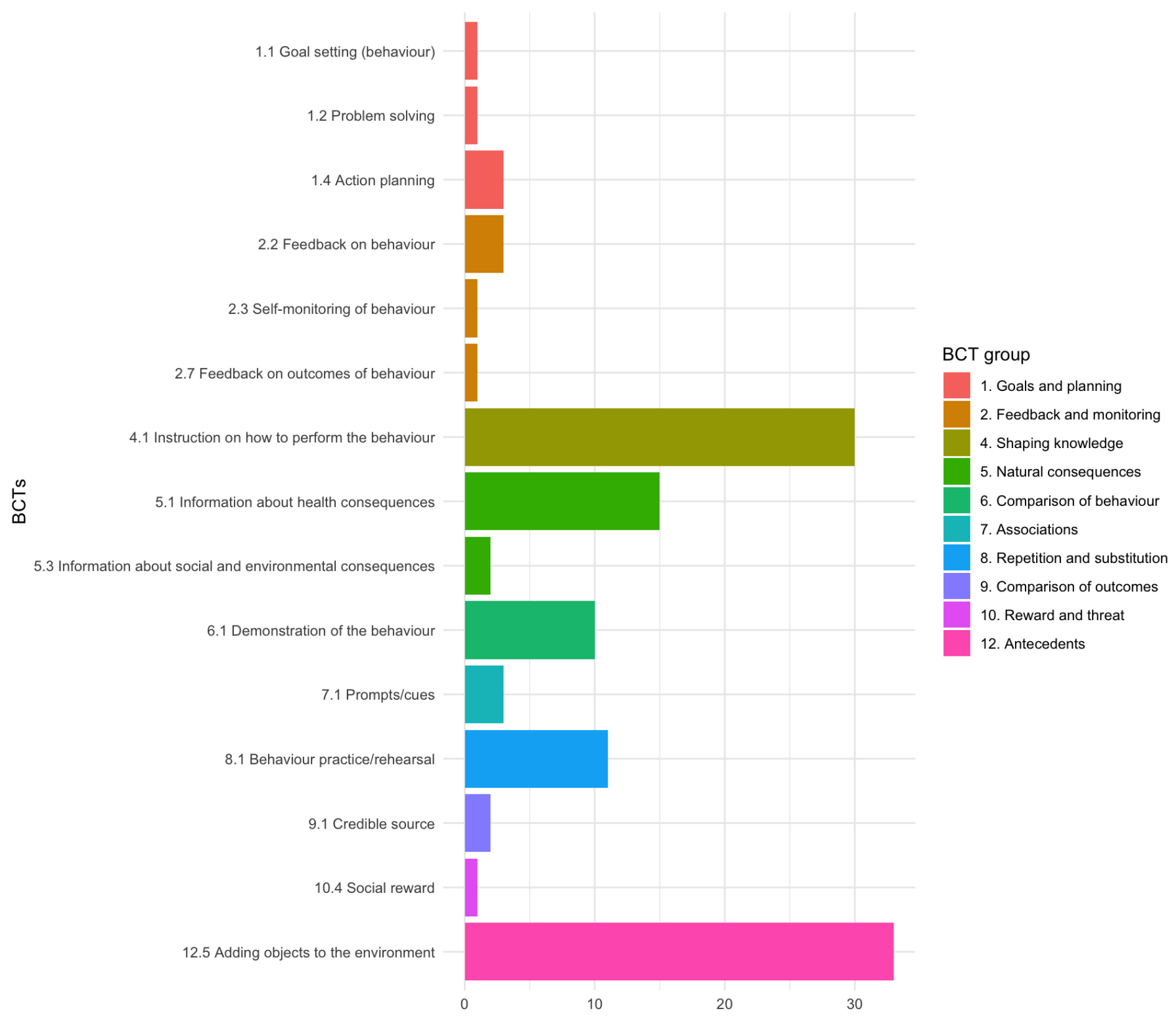

Figure 3. Frequencies of behaviour change techniques (BCTs) coded in included interventions.

\section{Acceptability}

Fourteen studies reported on the acceptability of interventions. Indicators assessed included mask comfort $38,52,53,59,66,70$, skin problems/irritation ${ }^{41,60,62,63,65}$, adverse events ${ }^{49}$, liking/positive impressions ${ }^{36}$ and ease of understanding ${ }^{35}$, with a small number of participants experiencing discomfort or irritation in the majority of studies that reported these outcomes (see Table 4).

\section{Practicability}


One study ${ }^{59}$ considered the practicability of scaling up mask fit testing outside the study setting and decided against including routine fit testing as part of the intervention (see Table 4).

\section{Effectiveness}

Studies relied on self-report $(25 / 39 ; 64 \%)$, direct observation $(7 / 39 ; 18 \%)$, a combination of self-report and direct observation $(5 / 39 ; 13 \%)$ or photos/video $(2 / 39 ; 5 \%)$ to examine intervention effectiveness (see Table 4). Outcome variables were heterogeneous across studies (e.g. the frequency or amount of hand sanitizer/soap use per day, the rate of compliance with hand hygiene, or the rate of compliance with face mask use).

\section{Hand hygiene}

Overall, the 30 studies pertaining to hand hygiene behaviours (including hand washing and/or hand sanitizer/soap use) had positive results, with 19 studies reporting positive effects $34-36,38,40,42,44,47,49-52,55-57,64,71,72,74$, three studies reporting negative effects ${ }^{42,44,74}$, six studies reporting no difference $38,39,43,45,48,68$ and six studies with indeterminate results $^{41,54,58,60,67,69}$ (see Figure 4). It should be noted that some studies reported more than one hand hygiene outcome (e.g. hand washing and hand sanitizer use). 


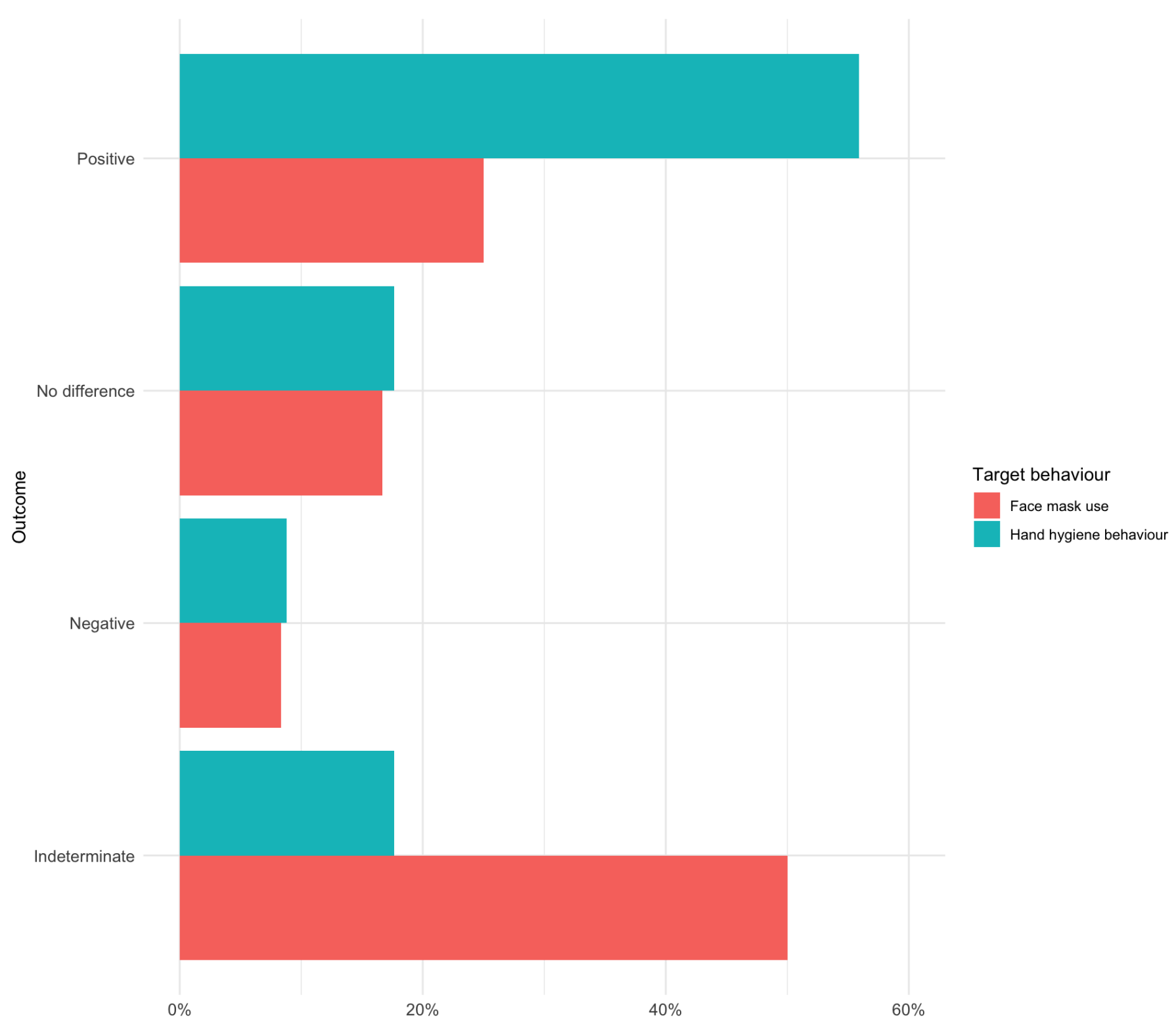

Figure 4. Proportions of reported effects (i.e. positive, negative, no difference or indeterminate) of interventions targeting hand hygiene behaviour and face mask use.

A random-effects meta-analysis $(k=6)$ found a medium, positive effect of interventions on the average frequency of hand hygiene behaviour, $d=0.62,95 \% \mathrm{Cl}=0.43-0.80, p<.001$ (see Figure 5). However, between-study heterogeneity was high $\left(1^{2}=81.2 \%\right)$. 


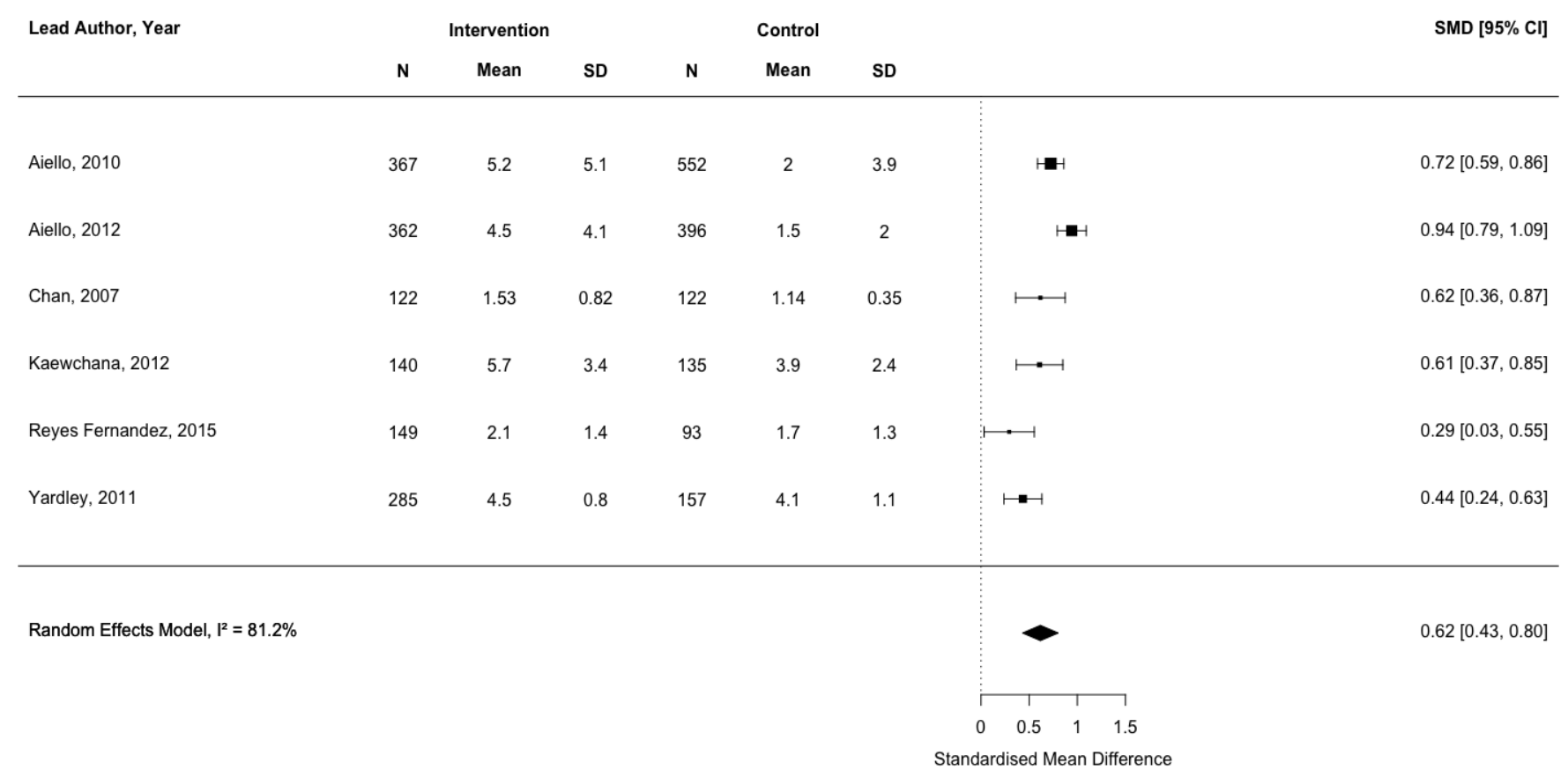

Figure 5. Forest plot for the standardised mean difference $(d)$ in the frequency of hand hygiene behaviour in intervention and control or pre- and post-study comparisons. The comparison in Chan ${ }^{50}$ pertains to a pre- and post-study comparison; the remaining studies were two- or three-arm RCTs.

\section{Face mask use}

Overall, the 12 studies pertaining to face mask use reported mixed results, with three studies reporting positive effects $^{48,49,66}$, two studies reporting no difference ${ }^{38,59}$, one study reporting negative effects ${ }^{37}$ and six studies with indeterminate results ${ }^{43,52,53,55,61,70}$ (see Figure 4).

A random-effects meta-analysis $(k=4)$ found a large, positive effect of interventions on the odds of compliance with face mask use, $\mathrm{OR}=4.14,95 \% \mathrm{Cl}=1.24-13.79, p<.001$ (see Figure 6). However, between-study heterogeneity was high $\left(\mathrm{I}^{2}=\right.$ $89.67 \%$ ) and the confidence interval for the pooled effect was wide. 


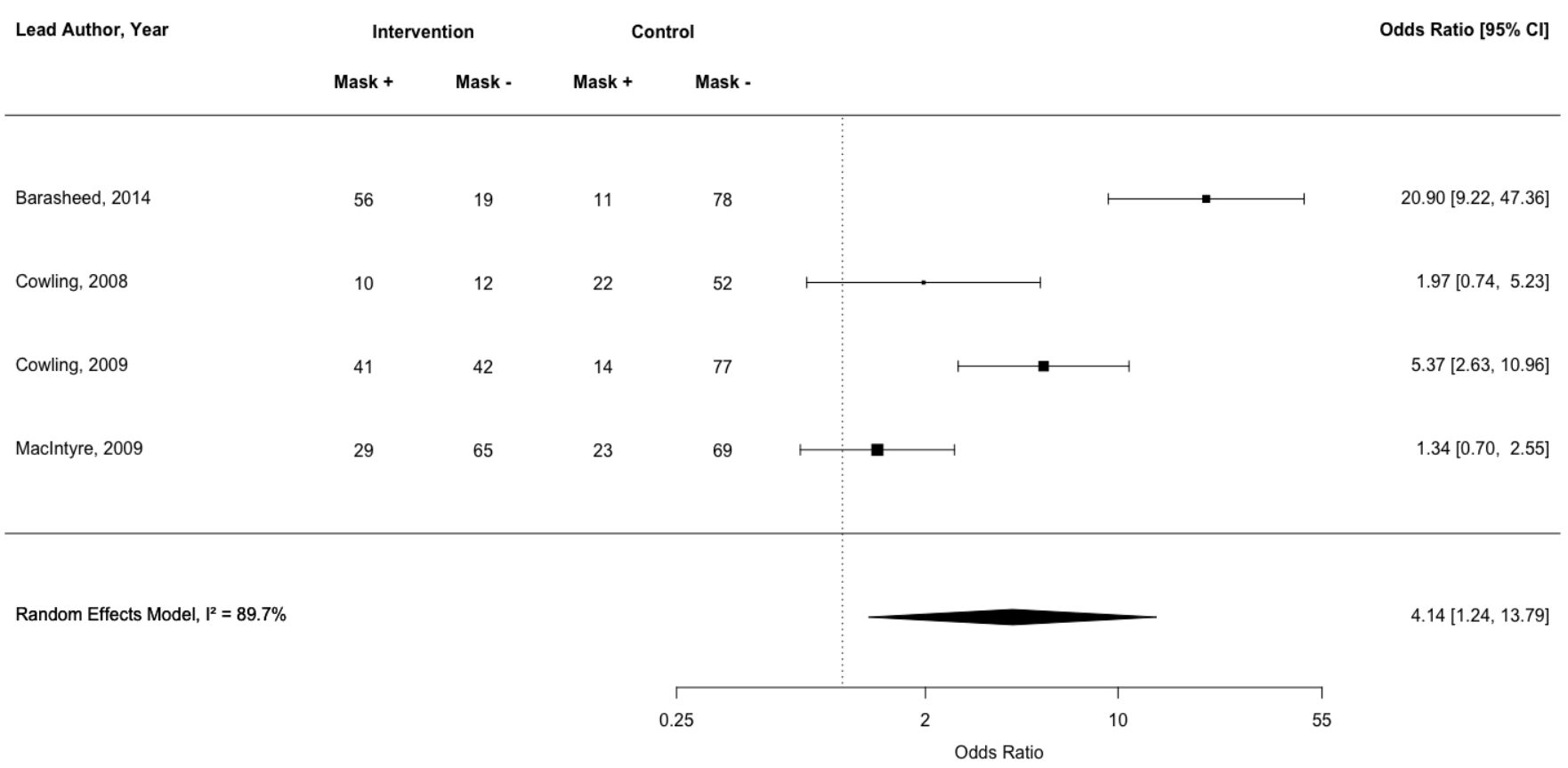

Figure 6. Forest plot for the odds of compliance with face mask use in intervention compared with control arms. The comparison in Maclntyre ${ }^{59}$ pertains to the surgical mask (intervention) versus the P2 mask arm (control) at the longest point of follow-up.

\section{Affordability}

Two studies considered the affordability of interventions, with one study discussing the cost of face masks ${ }^{61}$, which may act as a barrier for wider roll-out, and a second study ${ }^{71}$ reporting that although they wanted to provide hand hygiene products to all day care centre groups, they could only afford to do so for a maximum of two groups per centre due to budget restrictions, thus indicating that the selected intervention was not affordable at scale (see Table 4).

\section{Spill-over effects}

Three studies reported on secondary behaviour change (i.e. positive or negative spill-over to other behaviours), with one study ${ }^{71}$ assessing teachers' supervision of children's hand washing (in addition to their own hand washing), a second ${ }^{42}$ assessing the impact of the intervention on military leaders' hand sanitizer use (in addition to trainees') and a third 44 examining multiple hand hygiene behaviours in the same group of participants. The first study reported no change, the second a significant increase in hand sanitizer use and the third a negative impact on hand washing with soap (see Table 4). 


\section{Equity}

Four studies reported on the equity of interventions, with one study ${ }^{64}$ reporting that the intervention was equally effective for participants from high and low socioeconomic status groups and three studies ${ }^{44,46,62}$ reporting differential intervention effectiveness by educational attainment, parental income or ethnicity, with better outcomes reported in those with high educational attainment, high parental income and from a Black ethnic background (see Table 4).

\section{Quality of included studies}

One study received an overall rating of 'low risk of bias', with 16 studies rated as 'some concern', 18 as 'high risk of bias' and for four studies, a rating could not be applied (see Table 5).

Table 2. Characteristics of included studies.

\begin{tabular}{|c|c|c|c|c|c|c|c|c|c|c|}
\hline $\begin{array}{l}\text { Lead author } \\
\text { (year) }\end{array}$ & Country & $\begin{array}{l}\text { Target } \\
\text { behaviour(s) }\end{array}$ & $\begin{array}{l}\text { Study } \\
\text { design }\end{array}$ & Population & Sample size & $\begin{array}{l}\text { Mean age } \\
(S D)\end{array}$ & $\begin{array}{l}\% \\
\text { Female }\end{array}$ & $\begin{array}{l}\text { \% Post-16 } \\
\text { educational } \\
\text { qualifications }\end{array}$ & Setting & $\begin{array}{l}\text { Recri } \\
\text { strate }\end{array}$ \\
\hline 1) Aiello (2010) & United States & $\begin{array}{l}\text { Face mask } \\
\text { use and } \\
\text { hand } \\
\text { hygiene }\end{array}$ & $\begin{array}{l}\text { Three-arm, } \\
\text { cluster RCT }\end{array}$ & $\begin{array}{l}\text { University } \\
\text { students in } \\
\text { residence } \\
\text { halls }\end{array}$ & $\begin{array}{l}1297 \text { (face } \\
\text { mask + } \\
\text { hand } \\
\text { hygiene = } \\
367 ; \text { face } \\
\text { mask = } 378 ; \\
\text { control = } \\
552 \text { ) }\end{array}$ & $18.7(0.8)$ & $66 \%$ & $100 \%$ & $\begin{array}{l}\text { University } \\
\text { residence } \\
\text { halls }\end{array}$ & Not $r \epsilon$ \\
\hline 2) Aiello (2012) & United States & $\begin{array}{l}\text { Face mask } \\
\text { use and } \\
\text { hand } \\
\text { hygiene }\end{array}$ & $\begin{array}{l}\text { Three-arm, } \\
\text { cluster RCT }\end{array}$ & $\begin{array}{l}\text { University } \\
\text { students in } \\
\text { residence } \\
\text { halls }\end{array}$ & $\begin{array}{l}1178 \text { (face } \\
\text { mask + } \\
\text { hand } \\
\text { hygiene = } \\
362 ; \text { face } \\
\text { mask = 420; } \\
\text { control = } \\
396 \text { ) }\end{array}$ & $19.0(0.9)$ & $55 \%$ & $100 \%$ & $\begin{array}{l}\text { University } \\
\text { residence } \\
\text { halls }\end{array}$ & Not re \\
\hline $\begin{array}{l}\text { 3) Apisarnthanarak } \\
\text { (2009) }\end{array}$ & Thailand & $\begin{array}{l}\text { Hand } \\
\text { hygiene }\end{array}$ & $\begin{array}{l}\text { Single arm, } \\
\text { pre- and } \\
\text { post- } \\
\text { intervention } \\
\text { study }\end{array}$ & $\begin{array}{l}\text { Preschool } \\
\text { children }\end{array}$ & 240 & $5.0(1.7)$ & $49 \%$ & $0 \%$ & $\begin{array}{l}\text { Private } \\
\text { kindergarten }\end{array}$ & Not $r \epsilon$ \\
\hline 4) Arbogast (2016) & United States & $\begin{array}{l}\text { Hand } \\
\text { hygiene }\end{array}$ & $\begin{array}{l}\text { Two-arm, } \\
\text { cluster RCT }\end{array}$ & $\begin{array}{l}\text { Office } \\
\text { workers }\end{array}$ & $\begin{array}{l}1386 \\
\text { (intervention } \\
=604 ; \\
\text { control = } \\
782 \text { ) }\end{array}$ & $47.0(0.4)$ & $78 \%$ & Not reported & $\begin{array}{l}\text { Office } \\
\text { buildings }\end{array}$ & $\begin{array}{l}\text { E-mai } \\
\text { emplc }\end{array}$ \\
\hline 5) Azman (2013) & United States & $\begin{array}{l}\text { Hand } \\
\text { hygiene }\end{array}$ & $\begin{array}{l}\text { Two-arm, } \\
\text { cluster RCT }\end{array}$ & Households & 3360 & $\begin{array}{l}\text { Not } \\
\text { reported }\end{array}$ & $\begin{array}{l}\text { Not } \\
\text { reported }\end{array}$ & $0 \%$ & Schools & Not re \\
\hline $\begin{array}{l}\text { 6) Azor-Martinez } \\
\text { (2016) }\end{array}$ & Spain & $\begin{array}{l}\text { Hand } \\
\text { hygiene }\end{array}$ & $\begin{array}{l}\text { Two-arm } \\
\text { RCT }\end{array}$ & $\begin{array}{l}\text { School } \\
\text { children }\end{array}$ & 1341 & $8.0(2.3)$ & $68 \%$ & $0 \%$ & Schools & Not re \\
\hline $\begin{array}{l}\text { 7) Azor-Martinez } \\
\text { (2018) }\end{array}$ & Spain & $\begin{array}{l}\text { Hand } \\
\text { hygiene }\end{array}$ & $\begin{array}{l}\text { Three-arm, } \\
\text { cluster RCT }\end{array}$ & $\begin{array}{l}\text { Households } \\
\text { with a child } \\
\text { at a day care } \\
\text { centre }\end{array}$ & 911 children & Unclear & Unclear & Unclear & $\begin{array}{l}\text { Day care } \\
\text { centres }\end{array}$ & Not $r \epsilon$ \\
\hline
\end{tabular}




\begin{tabular}{|c|c|c|c|c|c|c|c|c|c|c|}
\hline $\begin{array}{l}\text { 8) Barasheed } \\
\text { (2014) }\end{array}$ & $\begin{array}{l}\text { Australia/Saudi } \\
\text { Arabia }\end{array}$ & $\begin{array}{l}\text { Face mask } \\
\text { use }\end{array}$ & $\begin{array}{l}\text { Two-arm, } \\
\text { pilot RCT }\end{array}$ & Hajj pilgrims & 164 & $\begin{array}{l}\text { Not } \\
\text { reported }\end{array}$ & $\begin{array}{l}\text { Not } \\
\text { reported }\end{array}$ & Not reported & Not reported & $\begin{array}{l}\text { Study } \\
\text { broch } \\
\text { distrik } \\
\text { mosq } \\
\text { Islami } \\
\text { and } p \\
\text { semir } \\
\text { in hot } \\
\text { Mecci }\end{array}$ \\
\hline $\begin{array}{l}\text { 9) Bundgaard } \\
\text { (2020) }\end{array}$ & Denmark & $\begin{array}{l}\text { Face mask } \\
\text { use }\end{array}$ & $\begin{array}{l}\text { Two-arm } \\
\text { RCT }\end{array}$ & $\begin{array}{l}\text { Community- } \\
\text { dwelling } \\
\text { adults }\end{array}$ & 6304 & $\begin{array}{l}47.2 \\
(13.5)\end{array}$ & $64 \%$ & Not reported & $\begin{array}{l}\text { Participants' } \\
\text { own homes }\end{array}$ & $\begin{array}{l}\text { Medie } \\
\text { adver } \\
\text { and th } \\
\text { conta } \\
\text { privat } \\
\text { comp } \\
\text { public } \\
\text { organ }\end{array}$ \\
\hline 10) Canini (2010) & France & $\begin{array}{l}\text { Face mask } \\
\text { use }\end{array}$ & $\begin{array}{l}\text { Two-arm, } \\
\text { cluster RCT }\end{array}$ & $\begin{array}{l}\text { Households } \\
\text { in three } \\
\text { regions of } \\
\text { France with } \\
\text { an index } \\
\text { patient }\end{array}$ & 105 & $\begin{array}{l}26.5 \\
(16.0)\end{array}$ & $\begin{array}{l}\text { Not } \\
\text { reported }\end{array}$ & Not reported & GP offices & GPs \\
\hline 11) Chan (2007) & China & $\begin{array}{l}\text { Catching } \\
\text { droplets in } \\
\text { tissues and } \\
\text { hand } \\
\text { hygiene }\end{array}$ & $\begin{array}{l}\text { Single arm, } \\
\text { pre- and } \\
\text { post- } \\
\text { intervention } \\
\text { study }\end{array}$ & $\begin{array}{l}\text { Registered } \\
\text { members of } \\
\text { a } \\
\text { government } \\
\text { subsidised } \\
\text { social } \\
\text { service } \\
\text { centre }\end{array}$ & 122 & $\begin{array}{l}\text { Not } \\
\text { reported }\end{array}$ & $63 \%$ & $20 \%$ & $\begin{array}{l}\text { Participants' } \\
\text { own homes }\end{array}$ & $\begin{array}{l}\text { Telep } \\
\text { calls }\end{array}$ \\
\hline $\begin{array}{l}\text { 12) Cowling } \\
\text { (2008) }\end{array}$ & China & $\begin{array}{l}\text { Face mask } \\
\text { use and } \\
\text { hand } \\
\text { hygiene }\end{array}$ & $\begin{array}{l}\text { Three-arm, } \\
\text { cluster RCT }\end{array}$ & $\begin{array}{l}\text { Households } \\
\text { with an } \\
\text { index patient }\end{array}$ & 198 & $\begin{array}{l}\text { Not } \\
\text { reported }\end{array}$ & $56 \%$ & Not reported & $\begin{array}{l}\text { Participants' } \\
\text { own homes }\end{array}$ & $\begin{array}{l}\text { Outpe } \\
\text { clinics }\end{array}$ \\
\hline $\begin{array}{l}\text { 13) Cowling } \\
\text { (2009) }\end{array}$ & China & $\begin{array}{l}\text { Face mask } \\
\text { use and } \\
\text { hand } \\
\text { hygiene }\end{array}$ & $\begin{array}{l}\text { Three-arm, } \\
\text { cluster RCT }\end{array}$ & $\begin{array}{l}\text { Households } \\
\text { with an } \\
\text { index patient }\end{array}$ & $\begin{array}{l}407 \text { index } \\
\text { patients }\end{array}$ & $\begin{array}{l}\text { Not } \\
\text { reported }\end{array}$ & $51 \%$ & Not reported & $\begin{array}{l}\text { Participants' } \\
\text { own homes }\end{array}$ & $\begin{array}{l}\text { Outpe } \\
\text { clinics }\end{array}$ \\
\hline 14) Hübner (2010) & Germany & $\begin{array}{l}\text { Hand } \\
\text { hygiene }\end{array}$ & $\begin{array}{l}\text { Two-arm } \\
\text { RCT }\end{array}$ & $\begin{array}{l}\text { Office } \\
\text { workers }\end{array}$ & 134 & $44.6(-)$ & $86 \%$ & Not reported & $\begin{array}{l}\text { Office } \\
\text { buildings }\end{array}$ & E-mai \\
\hline $\begin{array}{l}\text { 15) Kaewchana } \\
\text { (2012) }\end{array}$ & Thailand & $\begin{array}{l}\text { Hand } \\
\text { hygiene }\end{array}$ & $\begin{array}{l}\text { Two-arm } \\
\text { RCT }\end{array}$ & $\begin{array}{l}\text { Households } \\
\text { with an } \\
\text { index } \\
\text { paediatric } \\
\text { patient }\end{array}$ & $\begin{array}{l}275 \\
\text { (frequency } \\
\text { assessment) } \\
+330 \\
\text { (quality } \\
\text { assessment) } \\
\text { households }\end{array}$ & $\begin{array}{l}34.7 \\
(13.8)\end{array}$ & $58 \%$ & Not reported & $\begin{array}{l}\text { Participants' } \\
\text { own homes }\end{array}$ & Not re \\
\hline 16) Koер (2016) & United States & $\begin{array}{l}\text { Hand } \\
\text { hygiene }\end{array}$ & $\begin{array}{l}\text { Two-arm, } \\
\text { non- } \\
\text { randomised } \\
\text { cohort study }\end{array}$ & $\begin{array}{l}\text { School } \\
\text { children }\end{array}$ & 260 & $\begin{array}{l}\text { Not } \\
\text { reported }\end{array}$ & $46 \%$ & $0 \%$ & Schools & Not $r \epsilon$ \\
\hline 17) Larson (2009) & United States & $\begin{array}{l}\text { Hand } \\
\text { hygiene }\end{array}$ & $\begin{array}{l}\text { Single arm, } \\
\text { pre- and } \\
\text { post- } \\
\text { intervention } \\
\text { study }\end{array}$ & Households & $\begin{array}{l}422 \\
\text { households }\end{array}$ & $\begin{array}{l}\text { Not } \\
\text { reported }\end{array}$ & $\begin{array}{l}\text { Not } \\
\text { reported }\end{array}$ & $60 \%$ & $\begin{array}{l}\text { Participants' } \\
\text { own homes }\end{array}$ & $\begin{array}{l}\text { Neigr } \\
\text { snowl } \\
\text { techn } \\
\text { (e.g. c } \\
\text { schoc }\end{array}$ \\
\hline 3) Larson (2010) & United States & $\begin{array}{l}\text { Face mask } \\
\text { use and }\end{array}$ & Three-arm & Households & 509 & Not & $52 \%$ & $54 \%$ & Participants' & $\begin{array}{l}\text { Neigr } \\
\text { snowl } \\
\text { techn }\end{array}$ \\
\hline
\end{tabular}




\begin{tabular}{|c|c|c|c|c|c|c|c|c|c|c|}
\hline$\cdots,-\cdots, \ldots, \cdots,-\cdots$, & -.............. & $\begin{array}{l}\text { hand } \\
\text { hygiene }\end{array}$ & $\mathrm{RCT}$ & 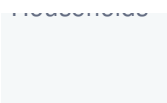 & $\cdots$ & reported & $-\cdots$ & $-\cdots$ & own homes & (e.g. c \\
\hline 19) Little (2015) & $\begin{array}{l}\text { United } \\
\text { Kingdom }\end{array}$ & $\begin{array}{l}\text { Hand } \\
\text { hygiene }\end{array}$ & $\begin{array}{l}\text { Two-arm } \\
\text { RCT }\end{array}$ & $\begin{array}{l}\text { Community- } \\
\text { dwelling } \\
\text { adults }\end{array}$ & 20,066 & $\begin{array}{l}56.6 \\
(13.6)\end{array}$ & $56 \%$ & Not reported & Online & $\begin{array}{l}\text { Maile } \\
\text { invita: } \\
\text { throus } \\
\text { surge }\end{array}$ \\
\hline $\begin{array}{l}\text { 21) Maclntyre } \\
\text { (2009) }\end{array}$ & Australia & $\begin{array}{l}\text { Face mask } \\
\text { use and } \\
\text { hand } \\
\text { hygiene }\end{array}$ & $\begin{array}{l}\text { Three-arm, } \\
\text { cluster RCT }\end{array}$ & $\begin{array}{l}\text { Households } \\
\text { with an } \\
\text { index } \\
\text { paediatric } \\
\text { patient }\end{array}$ & $\begin{array}{l}145 \text { families, } \\
290 \\
\text { caregivers }\end{array}$ & $\begin{array}{l}\text { Not } \\
\text { reported }\end{array}$ & $\begin{array}{l}\text { Not } \\
\text { reported }\end{array}$ & Not reported & Not reported & $\begin{array}{l}\text { Paedi } \\
\text { waitin }\end{array}$ \\
\hline 22) Mott (2007) & United States & $\begin{array}{l}\text { Hand } \\
\text { hygiene }\end{array}$ & $\begin{array}{l}\text { Three-arm, } \\
\text { non- } \\
\text { randomised } \\
\text { cohort study }\end{array}$ & $\begin{array}{l}\text { Military } \\
\text { trainees }\end{array}$ & 2728 & $20.2(-)$ & $0 \%$ & Unclear & $\begin{array}{l}\text { US army } \\
\text { training } \\
\text { centre }\end{array}$ & Not re \\
\hline $\begin{array}{l}\text { 23) Nandrup-Bus } \\
\text { (2009) }\end{array}$ & Denmark & $\begin{array}{l}\text { Hand } \\
\text { hygiene }\end{array}$ & $\begin{array}{l}\text { Two-arm, } \\
\text { cluster RCT }\end{array}$ & $\begin{array}{l}\text { School } \\
\text { children }\end{array}$ & 652 & $\begin{array}{l}\text { Not } \\
\text { reported }\end{array}$ & $51 \%$ & $0 \%$ & Schools & $\begin{array}{l}\text { Parer } \\
\text { sent v } \\
\text { study } \\
\text { inforn }\end{array}$ \\
\hline 24) Or (2020) & China & $\begin{array}{l}\text { Hand } \\
\text { hygiene }\end{array}$ & $\begin{array}{l}\text { Single arm, } \\
\text { pre- and } \\
\text { post- } \\
\text { intervention } \\
\text { study }\end{array}$ & $\begin{array}{l}\text { Households } \\
\text { with a child } \\
\text { in } \\
\text { kindergarten }\end{array}$ & $\begin{array}{l}58 \text { parents; } \\
60 \text { children }\end{array}$ & $\begin{array}{l}\text { Not } \\
\text { reported }\end{array}$ & $\begin{array}{l}\text { Parents } \\
=93 \% \\
\text { children } \\
=40 \%\end{array}$ & Not reported & Not reported & $\begin{array}{l}\text { Letter } \\
\text { princi } \\
\text { kinde } \\
\text { to invi } \\
\text { and c } \\
\text { partic }\end{array}$ \\
\hline 25) Ram (2015) & Bangladesh & $\begin{array}{l}\text { Hand } \\
\text { hygiene }\end{array}$ & $\begin{array}{l}\text { Two-arm, } \\
\text { cluster RCT }\end{array}$ & Households & $\begin{array}{l}377 \text { index } \\
\text { cases; } 384 \\
\text { household } \\
\text { compounds }\end{array}$ & $\begin{array}{l}121.2 \\
\text { months } \\
(181.7)\end{array}$ & $40 \%$ & Not reported & $\begin{array}{l}\text { Participants' } \\
\text { own homes }\end{array}$ & $\begin{array}{l}\text { Outpe } \\
\text { clinics }\end{array}$ \\
\hline $\begin{array}{l}\text { 26) Reyes } \\
\text { Fernández (2015) }\end{array}$ & Costa Rica & $\begin{array}{l}\text { Hand } \\
\text { hygiene }\end{array}$ & $\begin{array}{l}\text { Two-arm, } \\
\text { cluster RCT }\end{array}$ & $\begin{array}{l}\text { University } \\
\text { students }\end{array}$ & 242 & $21.0(3.9)$ & $61 \%$ & $100 \%$ & $\begin{array}{l}\text { University } \\
\text { classrooms }\end{array}$ & Not re \\
\hline 27) Roberts (2000) & Australia & $\begin{array}{l}\text { Hand } \\
\text { hygiene }\end{array}$ & $\begin{array}{l}\text { Two-arm, } \\
\text { cluster RCT }\end{array}$ & $\begin{array}{l}\text { Pre-school } \\
\text { children and } \\
\text { day care } \\
\text { staff }\end{array}$ & $\begin{array}{l}232 \\
\text { childcare } \\
\text { centres; } 558 \\
\text { children }\end{array}$ & $\begin{array}{l}\text { Not } \\
\text { reported }\end{array}$ & $\begin{array}{l}\text { Not } \\
\text { reported }\end{array}$ & $0 \%$ & $\begin{array}{l}\text { Childcare } \\
\text { centres }\end{array}$ & $\begin{array}{l}\text { Direc } \\
\text { care c } \\
\text { were }\end{array}$ \\
\hline $\begin{array}{l}\text { 28) Sandora } \\
\text { (2005) }\end{array}$ & United States & $\begin{array}{l}\text { Hand } \\
\text { hygiene }\end{array}$ & $\begin{array}{l}\text { Two-arm, } \\
\text { cluster RCT }\end{array}$ & $\begin{array}{l}\text { Households } \\
\text { with a child } \\
\text { at a day care } \\
\text { centre }\end{array}$ & 292 families & 36.7 (9.5) & $\begin{array}{l}\text { Not } \\
\text { reported }\end{array}$ & $91 \%$ & $\begin{array}{l}\text { Participants' } \\
\text { own homes }\end{array}$ & $\begin{array}{l}\text { Direc } \\
\text { care c } \\
\text { were } \\
\text { recrui } \\
\text { letter } \\
\text { to par }\end{array}$ \\
\hline $\begin{array}{l}\text { 29) Savolainen- } \\
\text { Kopra (2012) }\end{array}$ & Finland & $\begin{array}{l}\text { Hand } \\
\text { hygiene }\end{array}$ & $\begin{array}{l}\text { Three-arm, } \\
\text { cluster RCT }\end{array}$ & $\begin{array}{l}\text { Office } \\
\text { workers }\end{array}$ & 683 & $\begin{array}{l}\text { Not } \\
\text { reported }\end{array}$ & $\begin{array}{l}\text { Not } \\
\text { reported }\end{array}$ & Not reported & $\begin{array}{l}\text { Office } \\
\text { buildings }\end{array}$ & E-mai \\
\hline $\begin{array}{l}\text { 30) Simmerman } \\
\text { (2011) }\end{array}$ & Thailand & $\begin{array}{l}\text { Face mask } \\
\text { use and } \\
\text { hand } \\
\text { hygiene }\end{array}$ & $\begin{array}{l}\text { Three-arm } \\
\text { RCT }\end{array}$ & Households & $\begin{array}{l}442 \text { index } \\
\text { cases; } 1147 \\
\text { household } \\
\text { contacts }\end{array}$ & $\begin{array}{l}\text { Not } \\
\text { reported }\end{array}$ & $59 \%$ & Not reported & $\begin{array}{l}\text { Participants' } \\
\text { own homes }\end{array}$ & $\begin{array}{l}\text { Outpe } \\
\text { clinics }\end{array}$ \\
\hline $\begin{array}{l}\text { 31) Stebbins } \\
(2010)\end{array}$ & United States & $\begin{array}{l}\text { Hand } \\
\text { hygiene }\end{array}$ & $\begin{array}{l}\text { Two-arm, } \\
\text { cluster RCT }\end{array}$ & $\begin{array}{l}\text { School } \\
\text { children and } \\
\text { teachers }\end{array}$ & $\begin{array}{l}151 \\
\text { teachers }\end{array}$ & $\begin{array}{l}\text { Not } \\
\text { reported }\end{array}$ & $\begin{array}{l}\text { Not } \\
\text { reported }\end{array}$ & Not reported & Schools & Not re \\
\hline
\end{tabular}




\begin{tabular}{|c|c|c|c|c|c|c|c|c|c|c|}
\hline 33) Suess (2011) & Germany & $\begin{array}{l}\text { Face mask } \\
\text { use and } \\
\text { hand } \\
\text { hygiene }\end{array}$ & $\begin{array}{l}\text { Three-arm, } \\
\text { cluster RCT }\end{array}$ & $\begin{array}{l}\text { Households } \\
\text { with an } \\
\text { index patient } \\
\text { during the } \\
\text { H1N1 } \\
\text { pandemic }\end{array}$ & 147 & $\begin{array}{l}\text { Index } \\
\text { cases = } \\
7.9 \text { (3.3); } \\
\text { household } \\
\text { contacts = } \\
30.0 \\
(14.2)\end{array}$ & $52 \%$ & Not reported & $\begin{array}{l}\text { Participants' } \\
\text { own homes }\end{array}$ & $\begin{array}{l}\text { Outpe } \\
\text { clinics }\end{array}$ \\
\hline 34) Suess (2012) & Germany & $\begin{array}{l}\text { Face mask } \\
\text { use and } \\
\text { hand } \\
\text { hygiene }\end{array}$ & $\begin{array}{l}\text { Three-arm, } \\
\text { cluster RCT }\end{array}$ & $\begin{array}{l}\text { Households } \\
\text { with an } \\
\text { index patient } \\
\text { during the } \\
\text { H1N1 } \\
\text { pandemic }\end{array}$ & 302 & $\begin{array}{l}\text { Not } \\
\text { reported }\end{array}$ & $\begin{array}{l}\text { Not } \\
\text { reported }\end{array}$ & Not reported & $\begin{array}{l}\text { Participants' } \\
\text { own homes }\end{array}$ & $\begin{array}{l}\text { Outpe } \\
\text { clinics }\end{array}$ \\
\hline $\begin{array}{l}\text { 35) Updegraff } \\
\text { (2011) }\end{array}$ & United States & $\begin{array}{l}\text { Hand } \\
\text { hygiene }\end{array}$ & $\begin{array}{l}\text { Randomised } \\
\text { cross-over } \\
\text { study }\end{array}$ & $\begin{array}{l}\text { University } \\
\text { students and } \\
\text { staff }\end{array}$ & 65 units & $\begin{array}{l}\text { Not } \\
\text { reported }\end{array}$ & $\begin{array}{l}\text { Not } \\
\text { reported }\end{array}$ & Not reported & $\begin{array}{l}\text { Public areas } \\
\text { of a } \\
\text { university }\end{array}$ & $\begin{array}{l}\text { No ac } \\
\text { recrui }\end{array}$ \\
\hline 36) White (2003) & United States & $\begin{array}{l}\text { Hand } \\
\text { hygiene }\end{array}$ & $\begin{array}{l}\text { Two-arm, } \\
\text { cohort } \\
\text { study }^{*}\end{array}$ & $\begin{array}{l}\text { University } \\
\text { students }\end{array}$ & 430 & $18.3(0.7)$ & $62 \%$ & $0 \%$ & $\begin{array}{l}\text { University } \\
\text { residence } \\
\text { halls }\end{array}$ & Not re \\
\hline 37) Yardley (2011) & $\begin{array}{l}\text { United } \\
\text { Kingdom }\end{array}$ & $\begin{array}{l}\text { Hand } \\
\text { hygiene }\end{array}$ & $\begin{array}{l}\text { Two-arm, } \\
\text { pilot RCT }\end{array}$ & $\begin{array}{l}\text { Community- } \\
\text { dwelling } \\
\text { adults }\end{array}$ & 517 & $\begin{array}{l}49.8 \\
(11.4)\end{array}$ & $64 \%$ & Not reported & Online & $\begin{array}{l}\text { Maile } \\
\text { invita } \\
\text { GP si }\end{array}$ \\
\hline 38) Zomer (2016) & $\begin{array}{l}\text { The } \\
\text { Netherlands }\end{array}$ & $\begin{array}{l}\text { Hand } \\
\text { hygiene }\end{array}$ & $\begin{array}{l}\text { Two-arm, } \\
\text { cluster RCT }\end{array}$ & $\begin{array}{l}\text { Households } \\
\text { with a child } \\
\text { at a day care } \\
\text { centre }\end{array}$ & 71 centres & $\begin{array}{l}\text { Not } \\
\text { reported }\end{array}$ & $\begin{array}{l}\text { Not } \\
\text { reported }\end{array}$ & Not reported & Not reported & Not re \\
\hline 39) Öncü (2019) & Turkey & $\begin{array}{l}\text { Hand } \\
\text { hygiene }\end{array}$ & $\begin{array}{l}\text { Three-arm, } \\
\text { cluster RCT }\end{array}$ & $\begin{array}{l}\text { School } \\
\text { children }\end{array}$ & 96 & $9.2(1.0)$ & $54 \%$ & $0 \%$ & $\begin{array}{l}\text { School } \\
\text { laboratory }\end{array}$ & Not $r \epsilon$ \\
\hline & & & & & & & & & & - \\
\hline
\end{tabular}

Note. ${ }^{*}$ Unclear if randomised.

Table 3. Characteristics of interventions to change personal protective behaviours.

\begin{tabular}{|c|c|c|c|c|c|c|c|c|}
\hline $\begin{array}{l}\text { Lead author } \\
\text { (year) }\end{array}$ & Comparator & Intervention & $\begin{array}{l}\text { Intervention } \\
\text { duration }\end{array}$ & $\begin{array}{l}\text { BCTs } \\
\text { (comparator } \\
\text { arm) }\end{array}$ & $\begin{array}{l}\text { BCTs } \\
\text { (intervention } \\
\text { arm) }\end{array}$ & $\begin{array}{l}\text { Intervention } \\
\text { mode of } \\
\text { delivery }\end{array}$ & $\begin{array}{l}\text { Theoretical } \\
\text { mechanism(s) } \\
\text { of action of } \\
\text { the } \\
\text { intervention }\end{array}$ & $\begin{array}{l}\text { Incent } \\
\text { struct। } \\
\text { study } \\
\text { partici }\end{array}$ \\
\hline 1) Aiello (2010) & $\begin{array}{l}\text { Educational } \\
\text { materials }\end{array}$ & $\begin{array}{l}\text { The face mask group } \\
\text { received face masks; } \\
\text { written instructions on } \\
\text { how to use, store and } \\
\text { safely discard masks. } \\
\text { The face mask + hand } \\
\text { hygiene group also } \\
\text { received hand sanitizer }\end{array}$ & 6 weeks & $\begin{array}{l}\text { 4.1. Instruction } \\
\text { on how to } \\
\text { perform the } \\
\text { behaviour }\end{array}$ & $\begin{array}{l}\text { 4.1. Instruction on } \\
\text { how to perform the } \\
\text { behaviour; } 6.1 \text {. } \\
\text { Demonstration of } \\
\text { the behaviour; } 8.1 \text {. } \\
\text { Behaviour } \\
\text { practice/rehearsal; } \\
\text { 12.5. Adding } \\
\text { objects to the } \\
\text { environment }\end{array}$ & $\begin{array}{l}\text { Video link, } \\
\text { written } \\
\text { materials }\end{array}$ & Not reported & $\begin{array}{l}\text { Those } \\
\text { with in } \\
\text { like illn } \\
\text { were o } \\
\$ 25 \text { fol } \\
\text { providi } \\
\text { throat } \\
\text { specin }\end{array}$ \\
\hline 2) Aiello (2012) & $\begin{array}{l}\text { Educational } \\
\text { materials }\end{array}$ & $\begin{array}{l}\text { The face mask group } \\
\text { received face masks; } \\
\text { written instructions on } \\
\text { how to use, store and } \\
\text { safely discard masks. } \\
\text { The face mask + hand } \\
\text { hygiene group also } \\
\text { received hand sanitizer }\end{array}$ & 6 weeks & $\begin{array}{l}\text { 4.1. Instruction } \\
\text { on how to } \\
\text { perform the } \\
\text { behaviour }\end{array}$ & $\begin{array}{l}\text { 4.1. Instruction on } \\
\text { how to perform the } \\
\text { behaviour; } 8.1 \text {. } \\
\text { Behaviour } \\
\text { practice/rehearsal; } \\
\text { 12.5. Adding } \\
\text { objects to the } \\
\text { environment }\end{array}$ & $\begin{array}{l}\text { Written } \\
\text { materials }\end{array}$ & Not reported & Not rer \\
\hline & & & & & r.... & & & \\
\hline
\end{tabular}


3) Apisarnthanarak (2009)

$\begin{array}{ll}\begin{array}{ll}\text { An educational } \\ \text { video about hand }\end{array} & \begin{array}{l}\text { Same as the control } \\ \text { group in addition to }\end{array} \\ \begin{array}{ll}\text { hygiene; soap } & \text { hand sanitizers being } \\ \text { and hand } & \text { provided in different } \\ \text { sanitizer provided } & \text { areas of the office } \\ \text { in toilets } & \text { building }\end{array}\end{array}$

5) Azman (2013) Hand sanitizer

6) Azor-Martinez (2016)

7) Azor-Martinez (2018)

8) Barasheed (2014)
NA

Children, teachers and parents received hand hygiene (including sanitizer) education via cartoons and

workshops. A single dispenser of alcoholbased hand rub was placed in each nursery room

Same as control group in addition to a live demonstration of hand washing behaviour. Information about hand hygiene was sent home

Handwashing workshop; hand hygiene practices were periodically reinforced

No intervention in the classroom younger children were supervised during hand hygiene procedures; provision of hand sanitizer

Hand hygiene workshop with instruction on how to correctly perform the behaviour; use of stories, songs, posters; provision of hand sanitizer in one group and liquid soap in the other group; written materials on hand hygiene

In addition to the general hygiene information, face masks and written and verbal instructions on how to use these were provided

\section{Instructions to wear a}

2.2 reeaback on

behaviour; 2.7

Feedback on

outcomes of

behaviour; 4.1 .

Instruction on how Face-to-face

to perform the workshop,

behaviour; 6.1. cartoons, Not reported Not rer

Demonstration of written

the behaviour; 8.1. materials

Behaviour

practice/rehearsal;

12.5. Adding

objects to the

environment

4.1. Instruction on how to perform the

13.5 months behaviour; 12.5. Adding objects to the environment

4.1. Instruction on how to perform the behaviour; 6.1

Demonstration of the behaviour;

12.5. Adding

objects to the environment

4.1. Instruction on how to perform the behaviour; 6.1 .

Face-to-face

12.5. Adding

Not reported objects to the Demonstration of the behaviour; workshop, environment

12.5. Adding objects to the environment

4.1. Instruction on how to perform the behaviour; 6.1.

Demonstration of

the behaviour; 8.1.

Behaviour

Face-to-face

8 months NA

12.5. Adding

objects to the environment

\subsection{Instruction on}

how to perform the

behaviour; 6.1.

Demonstration of Face-to-face

the behaviour; 8.1. workshop,

Behaviour written

practice/rehearsal; materials

12.5. Adding

objects to the

environment

4.1. Instruction on how to perform the behaviour; 12.5 .

Adding objects to the environment

Face-to-face instructions, written Not reported Not rer materials

25 'we

points'

were 0

to emp

who

comple

both th

baselir

post-st

survey practice/rehearsal;

mask when outside the 


\begin{tabular}{|c|c|c|c|c|c|c|c|c|}
\hline $\begin{array}{l}\text { 9) Bundgaard } \\
\text { (2020) }\end{array}$ & $\begin{array}{l}\text { encouraging } \\
\text { participants to } \\
\text { follow current } \\
\text { COVID-19 } \\
\text { recommendations }\end{array}$ & $\begin{array}{l}\text { home during the next } \\
\text { month; provision of } 50 \\
\text { three-layer, disposable, } \\
\text { surgical face masks with } \\
\text { ear loops }\end{array}$ & 1 month & NA & $\begin{array}{l}\text { how to perform the } \\
\text { behaviour; } 12.5 \\
\text { Adding objects to } \\
\text { the environment }\end{array}$ & $\begin{array}{l}\text { Written } \\
\text { materials }\end{array}$ & Not reported & Not rer \\
\hline 10) Canini (2010) & No intervention & $\begin{array}{l}\text { Provision of face masks } \\
\text { and demonstration of } \\
\text { how to use them }\end{array}$ & 3 weeks & NA & $\begin{array}{l}6.1 . \\
\text { Demonstration of } \\
\text { the behaviour; } \\
\text { 12.5. Adding } \\
\text { objects to the } \\
\text { environment }\end{array}$ & $\begin{array}{l}\text { Face-to-face } \\
\text { instructions }\end{array}$ & Not reported & Not rer \\
\hline 11) Chan (2007) & NA & Health education & 7 days & NA & Not reported & $\begin{array}{l}\text { Telephone } \\
\text { calls with } \\
\text { trained } \\
\text { nursing } \\
\text { students }\end{array}$ & Not reported & Not rer \\
\hline $\begin{array}{l}\text { 12) Cowling } \\
(2008)\end{array}$ & $\begin{array}{l}\text { No intervention } \\
\text { (education about } \\
\text { healthy diet and } \\
\text { lifestyle) }\end{array}$ & $\begin{array}{l}\text { The face mask group } \\
\text { received face masks, } \\
\text { information about the } \\
\text { efficacy of masks and } \\
\text { instruction on how to } \\
\text { use and safely dispose } \\
\text { of masks; the hand } \\
\text { hygiene group received } \\
\text { hand sanitizer, liquid } \\
\text { soap, information about } \\
\text { the efficacy of hand } \\
\text { hygiene and } \\
\text { demonstration of hand } \\
\text { hygiene behaviour }\end{array}$ & 9 days & NA & $\begin{array}{l}\text { 4.1. Instruction on } \\
\text { how to perform the } \\
\text { behaviour; } 5.1 \text {. } \\
\text { Information about } \\
\text { health } \\
\text { consequences; } \\
6.1 \text {. } \\
\text { Demonstration of } \\
\text { the behaviour; } \\
\text { 12.5. Adding } \\
\text { objects to the } \\
\text { environment; }\end{array}$ & Not reported & Not reported & $\begin{array}{l}\text { At the 1 } \\
\text { home I } \\
\text { housel } \\
\text { were } \\
\text { reimbu } \\
\text { their } \\
\text { particir } \\
\text { with a } \\
\text { supern } \\
\text { vouch } \\
\text { approx } \\
\text { US \$21 }\end{array}$ \\
\hline 14) Hübner (2010) & No intervention & $\begin{array}{l}\text { Provision of hand } \\
\text { sanitizer and instruction } \\
\text { on how and when to use } \\
\text { it at work }\end{array}$ & 12 months & NA & $\begin{array}{l}\text { 4.1. Instruction on } \\
\text { how to perform the } \\
\text { behaviour; } 12.5 \text {. } \\
\text { Adding objects to } \\
\text { the environment }\end{array}$ & Not reported & Not reported & Not rer \\
\hline $\begin{array}{l}\text { 15) Kaewchana } \\
\text { (2012) }\end{array}$ & $\begin{array}{l}\text { 30-minute routine } \\
\text { health education } \\
\text { on influenza } \\
\text { infection, } \\
\text { nutrition, physical } \\
\text { activity, and } \\
\text { smoking } \\
\text { cessation }\end{array}$ & $\begin{array}{l}\text { 30-minute intensive } \\
\text { hand washing } \\
\text { education; individual } \\
\text { training on hand } \\
\text { washing; provision of } \\
\text { liquid soap; self- } \\
\text { monitoring diary; written } \\
\text { materials on hand } \\
\text { washing techniques }\end{array}$ & $x^{2}$ & $\begin{array}{l}\text { 4.1. Instruction } \\
\text { on how to } \\
\text { perform the } \\
\text { behaviour; } \\
5.1 \text {. } \\
\text { Information } \\
\text { about health } \\
\text { consequences }\end{array}$ & $\begin{array}{l}\text { 2.3. Self- } \\
\text { monitoring of } \\
\text { behaviour; } 4.1 \text {. } \\
\text { Instruction on how } \\
\text { to perform the } \\
\text { behaviour; } 5.1 \text {. } \\
\text { Information about } \\
\text { health } \\
\text { consequences; } \\
\text { 5.3. Information } \\
\text { about social and } \\
\text { environmental } \\
\text { consequences; } \\
\text { 8.1. behaviour } \\
\text { practice/rehearsal; }\end{array}$ & 西 & Not reported & Not rer \\
\hline
\end{tabular}


16) Koep (2016) No intervention

17) Larson (2009) NA

Educational

18) Larson (2010) materials on infection control

19) Little (2015) No intervention

20) Liu (2019) NA

21) MacIntyre (2009)
Educational intervention with information about microorganisms; provision of liquid soap and hand sanitizer in toilets and classrooms

Educational intervention focused on infection control; question and answer fact sheets; two groups of households were randomised to receive hand sanitizer, face masks or both

The hand sanitizer group received education plus hand sanitizer; the hand sanitizer and face mask group received the same interventions plus face masks

Four weekly web-based sessions with new content focused on the role of hand washing, setting up a plan to wash hands, reinforcement of helpful attitudes and norms, addressing negative beliefs, tailored feedback and prompts to login to the website

Hand hygiene training and information booklet; provision of soap, towels, posters, stickers, books, memory games and diplomas

Information about

infection control; provision of either $\mathrm{P} 2$ or surgical face masks

In the PI group, hand sanitizer dispensers were installed throuahout the trainina
12.5. Adding

objects to the

environment

4.1. Instruction on how to perform the behaviour; 5.1 .

Information about Face-to-face

health

Fith trained

Unclear NA

consequences;

teachers

12.5. Adding

objects to the

environment

4.1. Instruction of

how to perform the

behaviour; 5.1

Information about

health

Face-to-face

2 to 20 NA

consequences;

with trained

8.1. Behaviour researchers

practice/rehearsal;

12.5. Adding

objects to the

environment

4.1. Instruction on

how to perform the

behaviour; 5.1 .

Information about

health

Face-to-face

19 months Not reported

consequences;

8.1. behaviour

with trained Not reported Not rer practice/rehearsal;

12.5. Adding

objects to the

environment

\subsection{Action}

planning;2.2.

Feedback on

behaviour; 4.1.

Instruction on how

to perform the

Website

Not reported

Not rer

behaviour; 7.1

Prompts/cues; 8.1.

Behaviour

practice/rehearsal

4.1 Instruction on how to perform the behaviour; 10.4

Social reward;

Knowledge, perceived susceptibility,

12.5 Adding

posters,

severity,

objects to the

stickers,

perceived

environment

books

behavioural

control

12.5 Adding

objects to the environment 
22) Mott (2007)

23) Nandrup-Bus (2009)

24) Or (2020)

25) Ram (2015)

No intervention

26) Reyes

Fernández (2015) sanitize hands after key events (e.g. coughing, sneezing)

NA

No intervention
Hand sanitizer and instructions to wash or

information about

infection control and

techniques; parents

attended a separate

session with similar

content

Household compounds were provided with a hand washing station (e.g. water container with a tap, soap);

information on infection control and skills training; cue cards placed in a common area in compound courtyards

Instructions on how and when to clean hands and a planning task to help students set action and coping plans

Staff received training in hand washing and were asked to teach the techniaues to the

4.1 Instruction on how to perform the

Face-to-face behaviour; 9.1

12.5 Adding

Not reported objects to the environment

Credible source;

12.5 Adding

objects to the

environment sessions,

posters
Not reported Not rer

\subsection{Instruction on how to perform the behaviour; 5.1 Information about health consequences}

\section{Face-to-face}

sessions, Not reported Notrer posters

\subsection{Instruction on how to perform the behaviour; 5.1 Information about health consequences}

Tailored; daily intervention visits until 10 days following the resolution of the index case patient's symptoms

One-off NA

\subsection{Instruction on} how to perform the behaviour; 5.1 Information about health consequences; 12.5 Adding objects to the environment
Face-to-face sessions with an infection control nurse
Knowledge, skills

Not rer sessions, cue cards

Not reported Not rer

1.2 Problem
solving; 1.4 Action
planning; 4.1
Instruction on how
to perform the
behaviour

A face-toface session with research assistants, pamphlets
Action control, coping Not rer planning 
27) Roberts (2000) No intervention

28) Sandora (2005)

29) SavolainenKopra (2012)

30) Simmerman (2011)

31) Stebbins (2010)

No intervention (nutritional, physical activity, and smoking cessation education)

(educational materials about healthy eating; participants were asked not to use hand sanitizer during the study period)

No intervention

in their care via

songs about hand

Not reported NA

washing to the melodies

of nursery rhymes;

training was reinforced

with fortnightly visits and

newsletters

Provision of alcohol-

based hand sanitizer;

educational materials

(e.g. fact sheets, games,

toys) about hand

hygiene

Both groups received information on infection control. In the soap and water group, toilets were equipped with liquid hand soap. In the hand sanitizer arm, toilets were equipped with both liquid hand soap and alcohol-based hand rub

The hand washing group received education, instruction on hand washing techniques and a hand washing kit with liquid hand soap. The hand washing + face mask group received the same interventions as the hand washing group in addition to paper surgical face masks, training on how to use them appropriately and information about benefits of use

Students and staff received training in hand hygiene behaviors; schools placed and maintained supplies of alcohol-

based hand sanitizer in Not reported NA all classrooms and common areas; parents and guardians received educational materials on hand hygiene and home isolation practices

A brief training video to promote more effective A brief training video on infection control and demonstration of

communication effective hand

32) Stedman- with health care

nroviders washing/gel techniques;

hand sanitizer and how to perform the

behaviour; 6.1

Demonstration of

the behaviour

Face-to-face

training

sessions,

Not reported

Not rer

newsletters

12.5 Adding

objects to the

Fact sheets, environment games, toys

Not rer
4.1 Instruction on how to perform the behaviour; 5.1 Information about health consequences; 12.5 Adding objects to the environment

4.1 Instruction on how to perform the behaviour; 5.1 Information about health

consequences;

12.5 Adding

objects to the

environment

4.1 Instruction on how to perform the behaviour; 5.1 Information about health consequences;

12.5 Adding objects to the environment
Not reported Not reported Not rer

Face-to-face

House were compe with approx US \$61 Thai b: with trained Not reported study nurses

Teach were o $\$ 5$ gift comple each $\mathrm{s}$

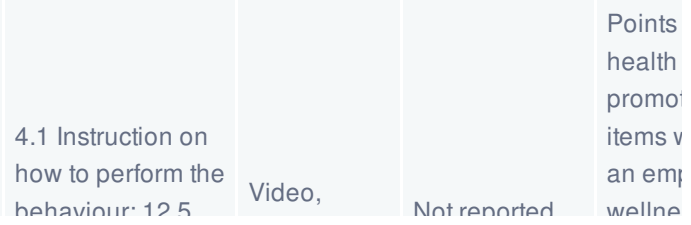




\section{branded key \\ chains, brochures \\ and posters with \\ information about \\ the programme \\ Educational \\ materials about \\ infection control \\ and}

33) Suess (2011) recommendation

to sleep in a

different room

than the index

patient

Educational

materials about

infection control

and

34) Suess (2012) recommendation

to sleep in a

different room

than the index

patient

35) Updegraff

(2011)

36) White (2003) No intervention motivational/educational

hand hygiene posters

were placed in break

rooms, kitchens and

conference rooms

Participants in the mask

+ hand hygiene and

mask groups were

provided with surgical

face masks with ear

loops and written

information on their

correct use; participants

in the mask + hand

hygiene group were

provided with alcohol-

based hand rub and

instructions on correct

use

Participants in the mask

+ hand hygiene and

mask groups were

provided with surgical

face masks with ear

loops and written

information on their

correct use; participants

in the mask + hand

hygiene group were

provided with alcohol-

based hand rub and

instructions on correct

use

Four signs were placed

above hand sanitizer

units. The perceived

susceptibility headline

read "Germs are out to

get you. Get them first!";

the social norms

headline read

"Everybody is doing it.

Are you?"; the gain-

framed headline read

"Stay healthy this

season. Sanitize your

hands"; and the loss-

framed headline read

"H1N1. Getting it is as

easy as passing me by."

Each sign contained a

"fact box" with more

detailed information

reinforcing the theme

Alcohol-gel dispensers

were installed in every

room, washroom, and

dining hall in residence

halls; a hand washing

message campaign was 10 weeks

implemented with

bulletin boards and

weekly messages to
Adding objects to

posters

progra

the environment

provid

each s

comple

how to perform the

Adding objects to

the environment

\section{visits by}

trained

study

personnel

4.1 Instruction on Written

how to perform the materials,

behaviour; 6.1

telephone,

Demonstration of face-to-face

the behaviour; visits by

Not reported

12.5 Adding

trained

objects to the

study

environment

personnel

5.1 Information

about health

consequences:

5.2 Information

12.5 Adding

about social and

environmental

Foam

Perceived

susceptibility,

objects to the

environment

consequences;

12.5 Adding

boards

social norms,

and attitudes

objects to the

environment

12.5 Adding

objects to the

Bulletin environment toward the

behavior
Not rer

$€ 150 \mathrm{fo}$

particir

Cash

incenti

totallin

maxim

$\$ 65$ 
37) Yardley (2011) No intervention

38) Zomer (2016) Usual care
Hand gel and 'photo shoots' before and after handwashing encourage hand

washing

Four weekly web-based sessions with

information about the medical team behind the advice (to enhance credibility), infection control, expert recommendations for hand washing frequency and technique, and instructions for picking up a free supply of hand gel from one's local GP practice; a hand washing plan to promote intention formation with situational cueing; tailored feedback to help participants improve their plan; reinforcement of positive attitudes and norms; addressing common negative beliefs; e-mail prompts to login to the website

Four components: 1) free hand hygiene products with refills for 6 months (e.g. soap, hand sanitizer);2) a

handwashing exercise with 'UV Glow Cream' and an information booklet with the training content; 3) two team training sessions focused on goal setting and identifying hand hygiene improvement activities; 4) posters and stickers placed in day care centres acting as reminders to practice hand hygiene

The first group received the same intervention as the control group plus information about getting rid of microbes on hands if washing hands 'properly'; the second group received the same interventions as the first plus a 30 minute hand hygiene educational and training session on types of microbes and diseases caused by microbes, in
1.4 Action

planning; 2.2

Feedback on

behaviour; 5.1

Information about

health

consequences:

7.1 Prompts/cues;

9.1 Credible

source; 12.5

Adding objects to

the environment

1.1 Goal setting

(behaviour); 7.1

Prompts/cues; 8.1

Behavioural

practice/rehearsal;

12.5 Adding

objects to the

environment

12.5 Adding

4 weeks objects to the environment

4.1 Instruction on how to perform the behaviour; 5.1 Information about health consequences;

8.1 Behavioural

Face-to-face session

Not reported

Not rer
Intention to wash hands, attitude, subjective norms, perceived behavioural control, perceived risk of infection 
addition to VWHU's $y$ -

stage handwashing

programme

Note. $\mathrm{NA}=$ not applicable.

Table 4. APEASE criteria, reach and engagement.

\begin{tabular}{|c|c|c|c|c|c|c|c|c|c|}
\hline $\begin{array}{l}\text { Lead author } \\
\text { (year) }\end{array}$ & Acceptability & Practicability & Affordability & $\begin{array}{l}\text { Spill-over } \\
\text { effects }\end{array}$ & Equity & Reach & Engagement & $\begin{array}{l}\text { How primary } \\
\text { outcome was } \\
\text { assessed }\end{array}$ & Effecti \\
\hline 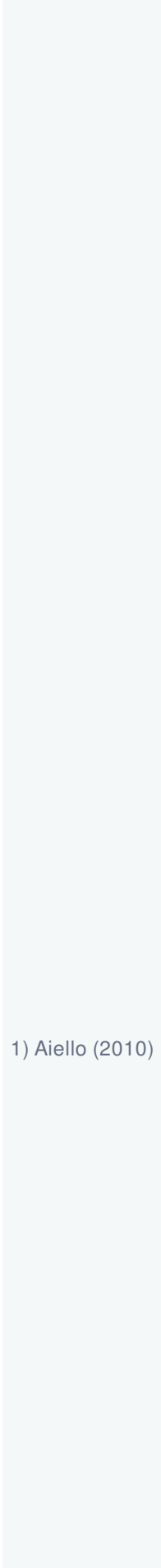 & 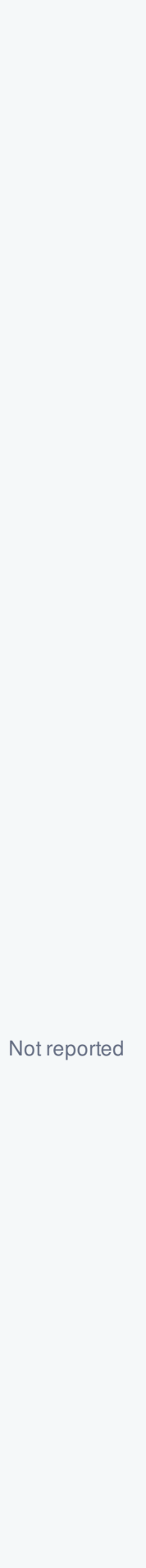 & 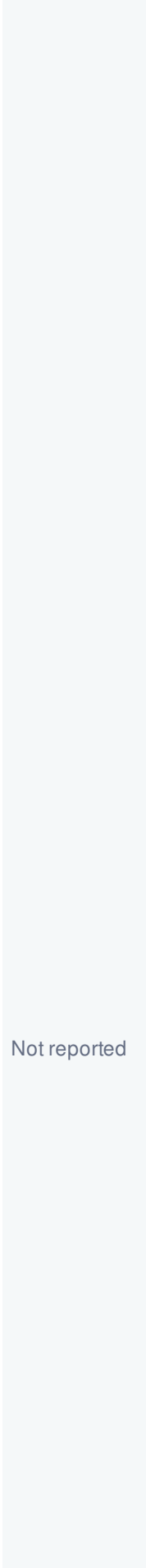 & 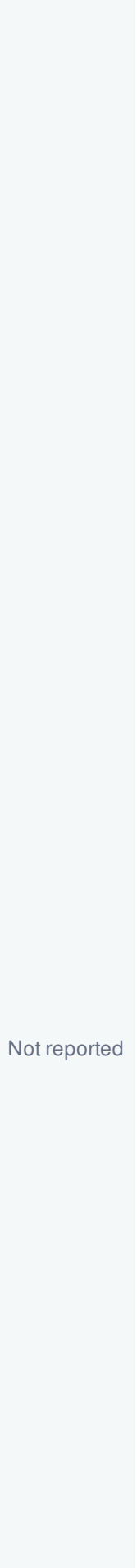 & 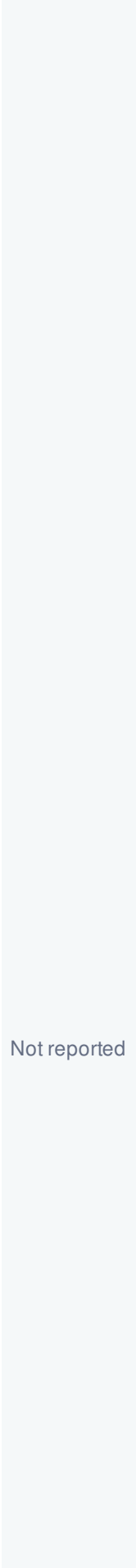 & 更 & $\begin{array}{l}\text { l/15 residence } \\
\text { halls were } \\
\text { included in the } \\
\text { study }\end{array}$ & Not reported & Self-report & 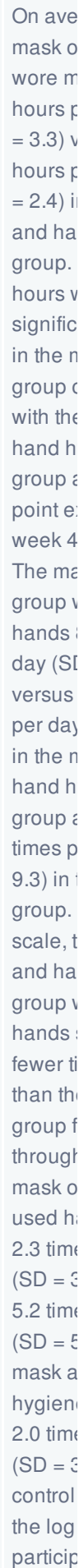 \\
\hline
\end{tabular}




\begin{tabular}{|c|c|c|c|c|c|c|c|c|c|}
\hline & & & & & & & & & $\begin{array}{l}\text { mask a } \\
\text { hygien } \\
\text { reporte } \\
\text { signific } \\
\text { use of I } \\
\text { sanitize } \\
\text { to the } n \\
\text { and col } \\
\text { at each } \\
\text { (p's }<.0 \text { | } \\
\text { were } n \\
\text { differer } \\
\text { betwee } \\
\text { only gr } \\
\text { control } \\
\text { frequer } \\
\text { sanitizt }\end{array}$ \\
\hline 2) Aiello (2012) & 要 & Not reported & Not reported & Not reported & Not reported & $\begin{array}{l}5 / 15 \text { residence } \\
\text { halls were } \\
\text { included in the } \\
\text { study }\end{array}$ & Not reported & $\begin{array}{l}\text { Self-report } \\
\text { and } \\
\text { observation of } \\
\text { mask wearing } \\
\text { by trained staff }\end{array}$ & 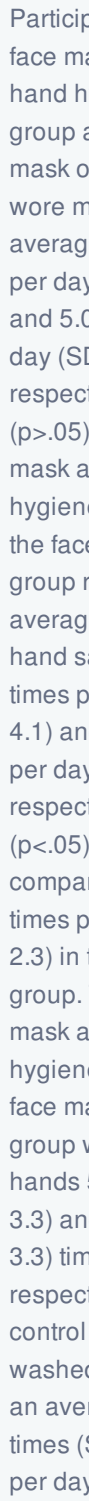 \\
\hline $\begin{array}{l}\text { 3) Apisarnthanarak } \\
\text { (2009) }\end{array}$ & Not reported & Not reported & 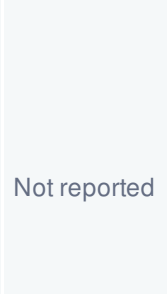 & Not reported & Not reported & Not reported & Not reported & $\begin{array}{l}\text { Observation of } \\
\text { hand hygiene } \\
\text { and cough } \\
\text { etiquette with } \\
\text { a Web camera } \\
1 \text { hour, twice }\end{array}$ & $\begin{array}{l}\text { The ob } \\
\text { of hanc } \\
\text { compli; } \\
\text { increas } \\
21 \% \text { ( } 5 \\
\text { period } \\
\text { (170/2 } \\
2 \text { (p<.0 }\end{array}$ \\
\hline
\end{tabular}


4) Arbogast (2016)

5) Azman (2013)

6) Azor-Martinez (2016)

7) Azor-Martinez (2018)

\begin{tabular}{|l|l} 
& \\
& \begin{tabular}{l} 
Employees in \\
the intervention \\
group were \\
significantly \\
more likely \\
than those in \\
the control \\
group to have \\
a positive \\
impression of \\
the programme \\
because of the \\
presence of \\
alcohol-based \\
hand sanitizers \\
in the \\
\hline workplace \\
(80\% vs $69 \%$, \\
p $<0.001)$. \\
$88 \%$ of \\
employees in \\
the intervention \\
group reported \\
liking the \\
products \\
provided
\end{tabular} \\
\hline 2016$)$
\end{tabular}

One child
showed
worsening of
existing atopic
dermatitis due
to hand
sanitizer gel
use and was
excluded from
the study

One child

showed

worsening of

existing

localized

atopic

dermatitis due

to hand

sanitizer gel

use and was

excluded from

the study

The most

commonly
$69 \%(1$

period

Self-re|

washin

improv

signific

time in

interve

$(p<.05)$

reporte

sanitize

1386/1609

employees

agreed to

participate

Not reported Self-report

every a

assess

includiı

eating,

sneeziı

coughi।

money

restroo

to their

interac

others '

sick ( $p$ '

Age and

ethnicity were

significant risk

Not reported Not reported Not reported

reported

influenza-like

illness $(p<.05)$

Predictors of a

lower rate of

absenteeism

due to

respiratory

illness

Not reported Not reported Not reported

included older

age, higher

parental

income, and

correct

handwashing

technique

$(p<.05)$

$1616 / 1640$

children were Not reported Self-report

Not rep randomised

Not reported Self-report

Not rep

\section{Not reported}

Not reported

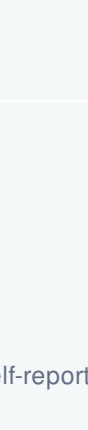

Not rep randomised 


\begin{tabular}{|c|c|c|c|c|c|}
\hline $\begin{array}{l}\text { 8) Barasheed } \\
\text { (2014) }\end{array}$ & $\begin{array}{l}\text { reported } \\
\text { reason for not } \\
\text { wearing face } \\
\text { masks was } \\
\text { discomfort } \\
\text { (15\% of } \\
\text { participants) }\end{array}$ & Not reported & Not reported & Not reported & Not reported \\
\hline $\begin{array}{l}\text { 9) Bundgaard } \\
(2020)\end{array}$ & Not reported & Not reported & $\begin{array}{l}\text { The authors } \\
\text { note that } \\
\text { costs and } \\
\text { availability } \\
\text { may reduce } \\
\text { the efficacy } \\
\text { of face } \\
\text { masks to } \\
\text { prevent } \\
\text { SARS-CoV- } \\
2 \text { infection }\end{array}$ & Not reported & Not reported \\
\hline
\end{tabular}

$17,258 / 6304$ of

those

responding to

recruitment

adverts were

randomised

95/105

randomised

households

completed the

study

$(33 \%)$

Children

wearing child

size face

masks reported

feeling pain

more

frequently than

those wearing

adult face

masks $(p=.036)$

11) Chan (2007)

Not reported Not reported Not reported Not reported

$182 / 295$

registered

members were

successfully

contacted;

$122 / 182$ took

part face mi

$76 \%(5$

interve।

and 12

the con

$\left(\mathrm{p}<.00^{-}\right.$

$46 \%$ of

in the it

arm wc

as recc

$47 \% \mathrm{pr}$

as recc

and $7 \%$

recomr

Particip

an ave

masks

weekd:

per we

Index $\mathrm{F}$

interve

houser

reporte

total of

7.2) $\mathrm{me}$

Self-report $\quad 4.0$ (SE

with an

use of:

1.3) me

and a $c$

use of:

2.7) ho

Signific
improv
observ
regard:
hands
sneeziı
( $\mathrm{M}_{\text {befor }}$
$=0.44$;
$1.65, \mathrm{~S}$
washin
with liq
(M $\mathrm{M}_{\text {befor }}$
$=0.35$;
$1.53, \mathrm{~S}$
and we
in publi
$1.31, \mathrm{~S}$ 


\begin{tabular}{|c|c|c|c|c|c|c|c|c|c|}
\hline & & & & & & & & & $\begin{array}{l}\mathrm{IV}_{\text {after }}= \\
1.12)(\mathrm{c} \\
\mathrm{p}^{\prime} \mathrm{s}<.0 \mathrm{OC}\end{array}$ \\
\hline $\begin{array}{l}\text { 12) Cowling } \\
\text { (2008) }\end{array}$ & 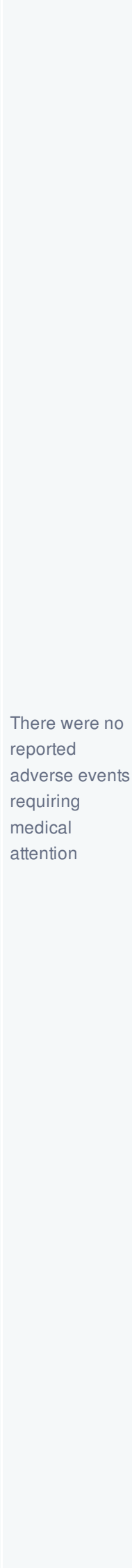 & Not reported & Not reported & Not reported & Not reported & $\begin{array}{l}\text { 198/944 index } \\
\text { patients were } \\
\text { randomised }\end{array}$ & Not reported & Self-report & 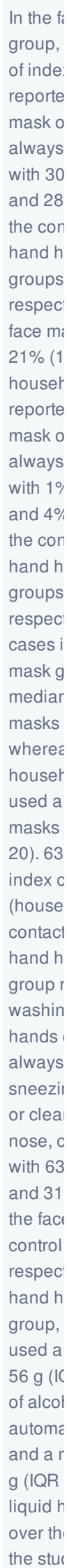 \\
\hline & & & & & & & & & $\begin{array}{l}\text { The pre } \\
\text { index c }\end{array}$ \\
\hline
\end{tabular}


13) Cowling (2009)

Not reported

Not reported
407/2750 index

patients and

their

households

were

randomised

$134 / 850$

participants

were

randomised wearin

masks

the con

(14/91)

hand $h$

group (

$49 \%$ (4

face $m$;

hand $h$

group.

proport

houser

wearin!

masks

(20/27s

control

(13/25;

hand $\mathrm{h}$

group;

(67/25£

mask a

hygienı

mediar

of liquic

hand $h$

group I

(42.4-1

78.9 (3

the fact

hand $h$

group.

(IQR) h

used in

hygien

$3.2 \mathrm{~g}(1$

index $\mathrm{C}$

$1.5 \mathrm{~g} \mathrm{(C}$

contact

compa|

g (0.7-5

cases c

(0.3-3.\&

in the f:

and ha

group

The $\mathrm{m} \epsilon$

disinfer

frequer

times d

3-5 tim

$60 \%$, a

daily in

On day

control

interve

reporte

2.4) an

3.4) ha

episod

respec

.001). 7

percen

particip

used si

increas 
16) Koep (2016) Not reported Not reported Not reported Not reported Not reported Not reported Not reported m

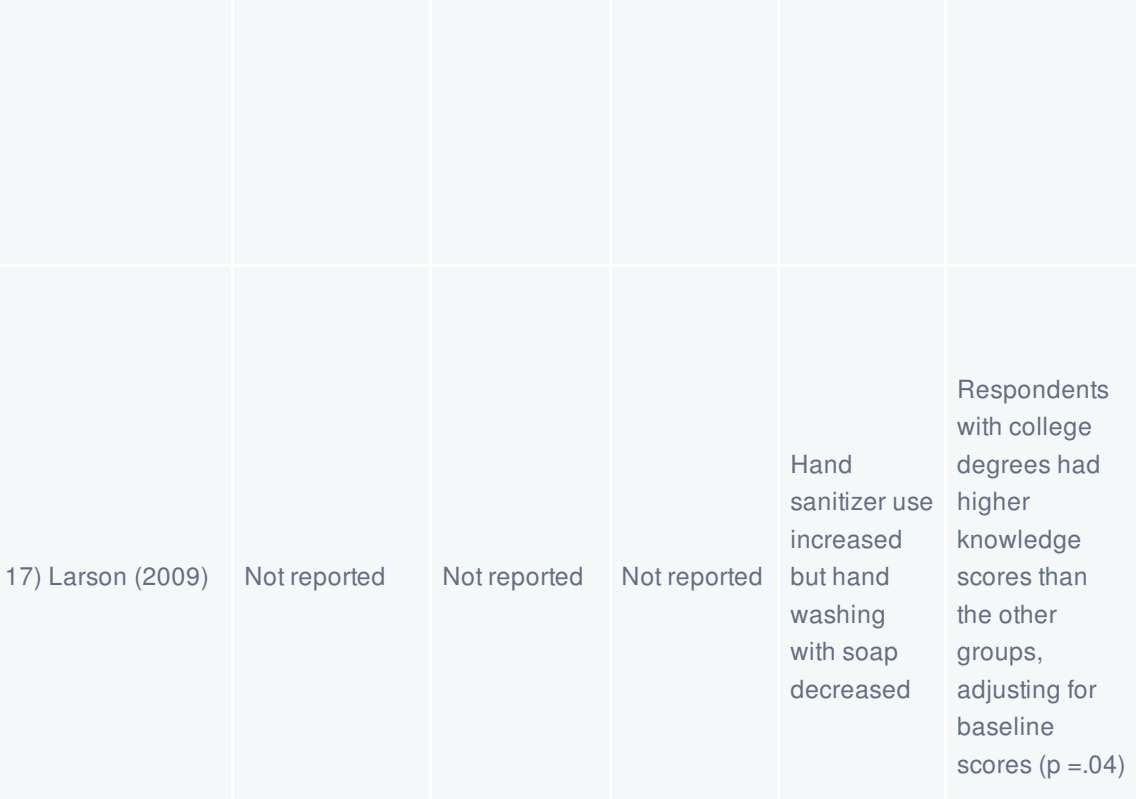

18) Larson (2010) Not reported

Not reported Not reported Not reported Not reported

$617 / 672$

households

that expressed

interest in

participation

met eligibility

criteria;

509/617

completed the

initial home

visit
$34 \%(5$

$88 \%(1$

$\left(\mathrm{p}<.00^{\circ}\right.$

No sigr

differer

soap cr

were ol

across

Self-report

(estima

and objective reporte

measure of sanitize

soap and increas

sanitizer use in the it

school

0.06 tin

studen

grades

(p's>. 0 :

A signi

greater

of partiı

reporte

Self-report

(questionnaire

administered

by trained

hand $\mathrm{s}$ :

the enc

(282/4:

Not reported researchers)

compa

baselin

measures of but a lo

hand sanitizer proport

using a

soap at

the stur

compa!

baselin

Particip

hand $\mathrm{s}$ :

group I

of 12.1

ounces

those it

sanitize

Self-report mask g

(questionnaire mean c

administered ounces

by trained $\quad(p=.36)$

$\begin{array}{ll}\text { Not reported researchers) houser } & \end{array}$

and objective case of

measures of reporte

hand sanitizer masks

and mask use hours $\mathrm{C}$

onset.

used $\mathrm{m}$

reporte

two

masks/

(range:

Minor self-

reported skin

irritation

increased

among those

who did not

report 
baseline.

19) Little (2015)

Among

Not reported

individuals

who had a skin

complaint at

baseline,

reported skin

complaints did

not significantly

increase over

time

20) Liu (2019)

\section{Not reported}

Not reported

There were no

significant

differences in

difficulties with

mask use

between the

P2 and

surgical mask

groups, but

$>50 \%$ reported

concerns, the

main one

being that

wearing a face

mask was

uncomfortable.

Other concerns

were that the

child did not

want the parent

wearing a

mask. Some

participants

mentioned that
¿U,Ubb/8U,48Y/

who received a

mailed

Not reported Self-report

Not rep

randomised

The av

reporte

compli;

hand $\mathrm{h}$

guideli।

signific

after th

interve

IQR = C

compa|

baselin

$=0.47$ )

$12 / 213$

kindergarten

clusters

Not reported Self-report

Teach€

signific

received the

intervention

$145 / 401$

families

assessed for

eligibility took

part

Not reported Self-report

On day

use, 38

the sur!

users a

(42/92)

mask u

that the

wearin!

"most c

time" $(\mathrm{k}$

Adhere

droppe

$(29 / 94)$

(23/92)

respec

5 of ma 


\begin{tabular}{|c|c|c|c|c|c|c|c|c|c|}
\hline & $\begin{array}{l}\text { the mask did } \\
\text { not fit well and } \\
\text { that it was not } \\
\text { practical to } \\
\text { wear at } \\
\text { mealtime or } \\
\text { while asleep }\end{array}$ & & & & & & & & \\
\hline 22) Mott (2007) & Not reported & Not reported & Not reported & $\begin{array}{l}\text { Hand } \\
\text { sanitizer use } \\
\text { increased in } \\
\text { leaders in } \\
\text { both } \\
\text { intervention } \\
\text { groups (from } \\
3.0 \text { to } 13.4 \\
\text { times/day } \\
\text { and from } 3.2 \\
\text { to } 4.7 \\
\text { times/day, } \\
\text { respectively) }\end{array}$ & Not reported & Not reported & Not reported & Self-report & $\begin{array}{l}\text { Post-in } \\
\text { there w } \\
\text { decrea } \\
\text { daily frt } \\
\text { hand w } \\
\text { the SI ç } \\
4.4 \text { to } 2 \\
\text { times/d } \\
\text { chang } \\
\text { group ( } \\
5.0 \text { tim } \\
\text { Hand s } \\
\text { increas } \\
\text { interve } \\
\text { (from } 3 \\
\text { times/d } \\
4.0 \text { to } 6 \\
\text { times/d } \\
\text { respec }\end{array}$ \\
\hline $\begin{array}{l}\text { 23) Nandrup-Bus } \\
\text { (2009) }\end{array}$ & $\begin{array}{l}\text { Three children } \\
\text { withdrew from } \\
\text { the intervention } \\
\text { arm due to skin } \\
\text { problems }\end{array}$ & Not reported & Not reported & Not reported & Not reported & Not reported & Not reported & $\begin{array}{l}\text { Objective } \\
\text { measure of } \\
\text { soap use }\end{array}$ & $\begin{array}{c}\text { School } \\
\text { shower } \\
\text { monthl } \\
\text { consun } \\
2.5 \text { litre } \\
\text { soap a } \\
\text { interve } \\
\text { During } \\
\text { interve } \\
\text { consun } \\
\text { increas } \\
\text { liquid s } \\
\text { accura } \\
\text { measul } \\
\text { not pos } \\
\text { soap w } \\
\text { continu } \\
\text { repleni } \\
\text { reliable } \\
\text { measul } \\
\text { soap c } \\
\text { in the c } \\
\text { school } \\
\text { availat }\end{array}$ \\
\hline \multirow[t]{2}{*}{ 24) Or (2020) } & \multirow[t]{2}{*}{ Not reported } & \multirow[t]{2}{*}{ Not reported } & \multirow[t]{2}{*}{ Not reported } & \multirow[t]{2}{*}{ Not reported } & \multirow[t]{2}{*}{ Not reported } & 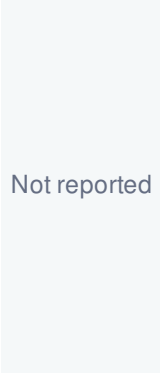 & \multirow[t]{2}{*}{ Not reported } & $\begin{array}{l}\text { Self-report } \\
\text { and } \\
\text { observation of } \\
\text { hand washing } \\
\text { with } \\
\text { fluorescent } \\
\text { stain gel and } \\
\text { photos }\end{array}$ & $\begin{array}{l}\text { After th } \\
\text { progral } \\
\text { percen } \\
\text { properl } \\
\text { areas C } \\
\text { hands i } \\
\text { signific } \\
\text { particul } \\
\text { (from } 0 \\
\text { p }<.001\end{array}$ \\
\hline & & & & & & $377 / 766$ & & $\begin{array}{l}\text { The } \\
\text { intervention } \\
\text { staff weighed } \\
\text { the soap each }\end{array}$ & $\begin{array}{l}\text { A medi } \\
\text { capita : } \\
\text { consun }\end{array}$ \\
\hline
\end{tabular}


25) Ram (2015) Not reported Not reported Not reported Not reported Not reported

26) Reyes Fernández (2015)

Not reported

Not reported

Not reported Not reported Not reported

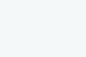

27)

27) Roberts (2000) Not reported

Not reported Not reported Not reported Not reported

28) Sandora

(2005)

29) SavolainenKopra (2012)

Forty-five

families

reported 112

adverse events

related to hand

sanitizer use.

Seventy-one

(63\%) of the

adverse events

were in relation

Other reported

adverse events

included

"stinging",

"smells bad",

"dislike it",

"allergic

reaction", and

"too slippery"

Not reported Not reported Not reported Not reported Not reported

eligible index Not reported
cases took part

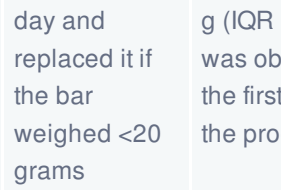

Self-re|

frequer

sanitize

day, me

a 5-poi

242/440

students

completed the

study

Not reported

Self-report

scale, i

both th,

(from N

0.9 to $\wedge$

1.3) an

interve।

(from N

1.3 to $\wedge$

1.4), $p=$

Hand $\mathrm{n}$

compli:

childre

divideo

groups

23/26 eligible

childcare

centres took

part

Observation

corresk

interve|

with a s

(53\%-6

centres

(70\%-7

centres

(>80\%:

compli:

292/647

families

assessed for

eligibility took

part

Not reported

Self-report

Primar

reporte

hand $\mathrm{s}$ :

a medi:

frequer

times/d

$683 / 1270$

employees eligibility took

part
The av amoun disinfer particip $g$ and $\epsilon$ active 
30) Simmerman (2011)

31) Stebbins (2010)

\begin{tabular}{|l|l|}
\hline Smith (2015) & \\
\hline 32) Stedman- & Not reported \\
\hline & \\
\hline & $\begin{array}{l}\text { The majority } \\
(60 \%) \text { of } \\
\text { participants did } \\
\text { not report any } \\
\text { problems when } \\
\text { wearing face } \\
\text { masks. Of } \\
\text { those who }\end{array}$ \\
\hline
\end{tabular}

$465 / 20,537$

paediatric

outpatients with Not reported Self-report influenza-like

illness took part

$151 / 167$

enrolled

teachers took part

Not reported Not reported Not reported Not reported
$324 / 1708$

enrolled office workers took part hand w

group $r$

washin

episod

compa|

times/d

hand w

face $\mathrm{mi}$

3.9 tim

control:

Particip

face m:

used al

12 mas

person

(media

$=7-16)$

reporte

face $\mathrm{m}$;

mediar

minute:

17-31:

wore th

for a m

(IQR =

minute:

which v

than ot

(media

= 9-26

cases t

(media

=4-19

sibling:

17; IQF

The prc

studen:

their he

than 3 .

day in 1

season

signific

in the it

arm (M

compa

control

3.4), $p=$

In both

interve

control

$>80 \%$ c

employ

reporte

sanitize

least 2!

time ( $p$ : 


\section{reported}

having

$81 \%$ of

removed their

and 71

masks in

transmission-

houser

prone

membe

situations,

'feeling hot'

was the main

combin

groups

mask 'c

'most o

reason. Other

when is

problems

room w

mentioned less

healthy

frequently were

$\begin{array}{ll}\text { 33) Suess (2011) } & \text { pain when } \\ \text { wearing the }\end{array}$

Not reported Not reported Not reported Not reported Not reported Not reported Self-report

person

mask and

shortness of

breath. The

majority of

adult

household

contacts in the

control and

intervention

groups

perceived

wearing face

masks as well

as intensified

hand hygiene

as an effective

means of

preventing

transmission of

influenza

The majority of

Not reported

Particir

cases ć

combin

mask $p$

group

washer

their he

signific

frequer

compal

those it

and $\mathrm{Co}$ ।

combin

$(p=.00$;

participants

$(62 \%)$ did not

report any

problems with

mask wearing.

This proportion

was

significantly

higher in adults

$(71 \%)$ than

children (50\%)

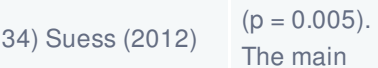

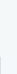

problem stated

by participants

(adults as well

as children)

was

"heat/humidity",

followed by

"pain" and

"shortness of

breath" when

wearing a face

Particip

mask g

mediar

(IQR =

masks

individı

Particip

mask p

group ı

mediar

The amount of (IQR =

remaining masks.

intervention of hanc

Not reported Not reported Not reported Not reported Not reported Not reported materials was disinfer

assessed at day wa

the end of the 4.1 in is

study period in the 2

2010/1

respec

numbe

disinfer

day wa

7.5 in h

contact

2009/1

2010/1

respec

All sign

signific

hand s: 
35) Updegraff

(2011)
There were no

significant

differences

between the

four signs in

how easy they

were to

understand.

The loss-

framed and

perceived

susceptibility

signs elicited

significantly

more negative

affect than the

gain-framed

and norms

signs (p's

$<.01)$.
Not reported Not reported Not reported Not reported Not reported

Notreported

ot reported

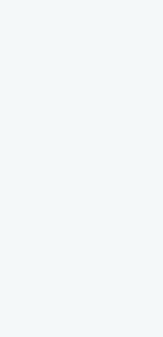

compal

no sign

but the'

equally

Dispen

gain-fré

had the

hand $\mathrm{s}$;

with 66

use tha

dispen:

signs (1

Loss-fr:

Grams of

sanitizer used

per day,

were a:

with a ?

increas

measured

with a digital

scale

compa

sign $(p$.

social r

$(44.3 \%$

and the

suscep

$(40.6 \%$

were a:

with so

lower it

usage ,

with the

framed

framed

both le

signific

use tha

(both $p$

Over th

the stur

produc

washer

$10.4 \%$

frequer

control

times/h

times/h

frequer

sanitize

also siç

greater

produc

times/h

times/h

Of the 324

participants

who were

randomly

assigned to

the

intervention,

251 (77.5\%)

Signific

differer

progressed

to the

second

session, 219

(67.6\%)

completed

th....

betwee

hand $w$

frequer

measul

point si

observi 


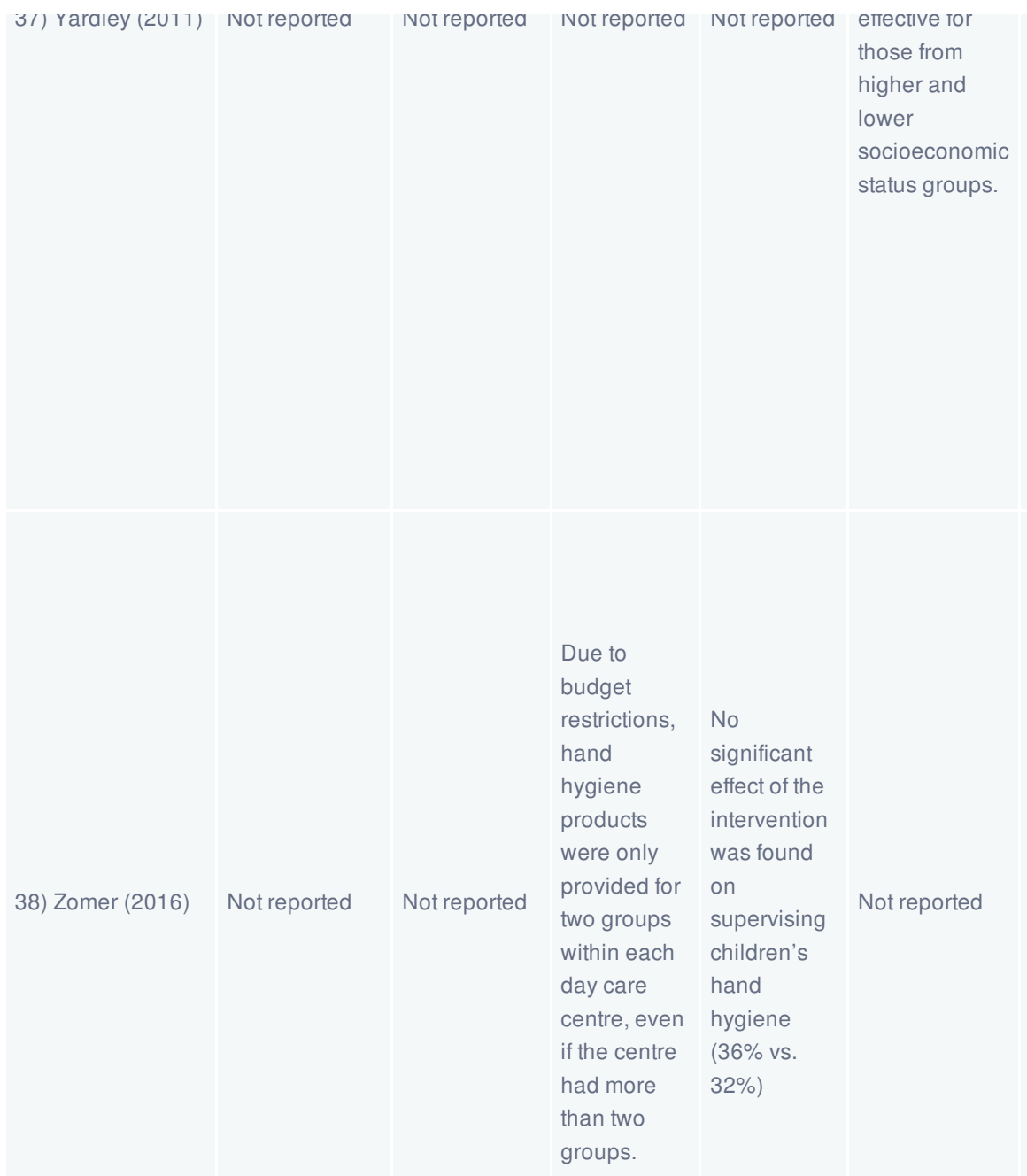

39) Öncü (2019) Not reported Not reported Not reported Not reported Not reported 96/552 children Not reported

\begin{tabular}{|c|c|c|c|}
\hline $\begin{array}{l}\text { Inose Invitea } \\
\text { participated }\end{array}$ & $\begin{array}{l}\text { tnree } \\
\text { sessions, } \\
\text { and } 188 \\
(58.0 \%) \\
\text { completed } \\
\text { all four } \\
\text { sessions. } \\
\text { The free } \\
\text { hand gel } \\
\text { was } \\
\text { collected by } \\
170 / 324 \\
\text { (52.5\%) } \\
\text { eligible } \\
\text { participants. }\end{array}$ & seıt-report & $\begin{array}{l}\text { weeks } \\
=0.9 \mathrm{v} \text { s } \\
\mathrm{SD}=0 \\
\mathrm{~d}=0.4 \\
\text { weeks } \\
=1.1 \mathrm{v} \text { ! } \\
\mathrm{SD}=0 \\
\mathrm{~d}=0.3\end{array}$ \\
\hline $\begin{array}{l}71 / 122 \text { day } \\
\text { care centres } \\
\text { participated }\end{array}$ & $\begin{array}{l}\text { Of } 274 \\
\text { caregivers, } \\
21 \% \\
(54 / 261) \\
\text { attended } \\
\text { none of the } \\
\text { training } \\
\text { sessions, } \\
25 \% \\
(66 / 261) \\
\text { attended one } \\
\text { training } \\
\text { session, } \\
29 \% \\
\text { (75/261) } \\
\text { attended two } \\
\text { training } \\
\text { sessions and } \\
25 \% \\
\text { (66/261) } \\
\text { attended all } \\
\text { three } \\
\text { sessions. }\end{array}$ & $\begin{array}{l}\text { Direct } \\
\text { observation } \\
\text { by trained } \\
\text { researchers }\end{array}$ & $\begin{array}{l}\text { Hand h } \\
\text { complii } \\
\text { interve } \\
\text { care ce } \\
66 \% \text { vs } \\
\text { control } \\
=6.33 \text {, } \\
3.71-1\end{array}$ \\
\hline
\end{tabular}

In the $\mathrm{n}$ intensil Photos interve examined by post-in researchers hand $\mathrm{w}$ not directly effectiv involved in the scores study signific all regi hands

Note. + = positive effect; - = negative effect; / = no change; @ = indeterminate; NA = not applicable.

Table 5. Quality appraisal.

\begin{tabular}{|c|c|c|c|c|c|c|}
\hline $\begin{array}{l}\text { Lead author } \\
\text { (year) }\end{array}$ & $\begin{array}{l}\text { 1) Bias arising from the } \\
\text { randomisation process }\end{array}$ & $\begin{array}{l}\text { 2) Bias due to deviations } \\
\text { from the intended } \\
\text { interventions }\end{array}$ & $\begin{array}{l}\text { 3) Bias due to } \\
\text { missing outcome } \\
\text { data }\end{array}$ & $\begin{array}{l}\text { 4) Bias in } \\
\text { measurement of } \\
\text { the outcome }\end{array}$ & $\begin{array}{l}\text { 5) Bias in selection } \\
\text { of the reported } \\
\text { result }\end{array}$ & $\begin{array}{l}\text { Overall } \\
\text { rating }\end{array}$ \\
\hline 1) Aiello (2010) & Some concerns & Low risk of bias & Some concerns & Low risk of bias & Low risk of bias & $\begin{array}{l}\text { Some } \\
\text { concerns }\end{array}$ \\
\hline 2) Aiello (2012) & Some concerns & Low risk of bias & Some concerns & Some concerns & Low risk of bias & $\begin{array}{l}\text { Some } \\
\text { concerns }\end{array}$ \\
\hline
\end{tabular}


3) Apisarnthanarak (2009)

NA

4) Arbogast (2016) Some concerns

5) Azman (2013) Some concerns

Some concerns

6) Azor-Martinez

(2016)

7) Azor-Martinez

(2018)

Some concerns

8) Barasheed

(2014)

9) Bundgaard

(2020)

10) Canini (2010) Some concerns

11) Chan (2007)

NA

12) Cowling

(2008)

13) Cowling

(2009)

14) Hübner (2010) Some concerns

15) Kaewchana

Some concerns

16) Koep (2016) Some concerns

17) Larson (2009) NA

18) Larson (2010) Some concerns

19) Little (2015) Some concerns

20) Liu (2019) NA

21) Maclntyre (2009)

22) Mott (2007)

23) Nandrup-Bus (2009)

24) Or (2020)

NA

25) Ram (2015) Some concerns

26) Reyes

Fernández (2015) Some concerns

27) Roberts (2000) Some concerns

28) Sandora

(2005)

Low risk of bias

29) Savolainen-
NA

Some concerns

Some concerns

Some concerns

Some concerns

Some concerns

Some concerns

Some concerns

Some concerns

Some concerns

Some concerns

Some concerns

Some concerns

Some concerns

Some concerns

Some concerns

Some concerns

NA

Some concerns

NA

Some concerns

NA

Some concerns

Some concerns

Some concerns

Some concerns
NA

NA

NA

NA

Low risk of bias

Low risk of bias

Some concerns

Some concerns

Some concerns

Low risk of bias

Low risk of bias

Some concerns

Some concerns

Some concerns

Some concerns

Low risk of bias

Low risk of bias

Some concerns

Some concerns

Low risk of bias

Some concerns

Some concerns

Low risk of bias

Some concerns

Some concerns

Some concerns

Some concerns

Some concerns

Some concerns

Low risk of bias

Some concerns

Low risk of bias

NA

NA

Low risk of bias

Some concerns

NA

NA

Some concerns

Some concerns

NA

NA

Low risk of bias

Low risk of bias

Some concerns

Some concerns

Some concerns

Low risk of bias

Some concerns
NA

Some concerns

Some

concerns

Some

concerns

High risk

of bias

Some

concerns

High risk

of bias

High risk

of bias

Some

concerns

High risk

of bias

Some

concerns

Some

concerns

High risk

of bias

High risk

of bias

High risk

of bias

High risk

of bias

High risk

of bias

High risk

of bias

NA

Some

concerns

NA

High risk of bias

NA

Some

concerns

High risk

of bias

High risk of bias

High risk of bias

High risk 


\begin{tabular}{|c|c|c|c|c|c|c|}
\hline Kopra (2012) & Some concerns & Some concerns & Some concerns & Some concerns & Low risk of bias & of bias \\
\hline $\begin{array}{l}\text { 30) Simmerman } \\
(2011)\end{array}$ & Low risk of bias & Low risk of bias & Some concerns & Some concerns & Low risk of bias & $\begin{array}{l}\text { Some } \\
\text { concerns }\end{array}$ \\
\hline $\begin{array}{l}\text { 31) Stebbins } \\
(2010)\end{array}$ & Some concerns & Some concerns & Low risk of bias & Some concerns & Some concerns & $\begin{array}{l}\text { High risk } \\
\text { of bias }\end{array}$ \\
\hline $\begin{array}{l}\text { 32) Stedman- } \\
\text { Smith (2015) }\end{array}$ & Low risk of bias & Low risk of bias & Some concerns & Some concerns & Some concerns & $\begin{array}{l}\text { Some } \\
\text { concerns }\end{array}$ \\
\hline 33) Suess (2011) & Low risk of bias & Low risk of bias & Some concerns & Some concerns & Some concerns & $\begin{array}{l}\text { Some } \\
\text { concerns }\end{array}$ \\
\hline 34) Suess (2012) & Low risk of bias & Some concerns & Some concerns & Some concerns & Some concerns & $\begin{array}{l}\text { High risk } \\
\text { of bias }\end{array}$ \\
\hline $\begin{array}{l}\text { 35) Updegraff } \\
\text { (2011) }\end{array}$ & Low risk of bias & Low risk of bias & Low risk of bias & Low risk of bias & Low risk of bias & $\begin{array}{l}\text { Low risk } \\
\text { of bias }\end{array}$ \\
\hline 36) White (2003) & High risk of bias & Low risk of bias & Some concerns & Some concerns & Some concerns & $\begin{array}{l}\text { High risk } \\
\text { of bias }\end{array}$ \\
\hline 37) Yardley (2011) & Low risk of bias & Low risk of bias & Some concerns & Some concerns & Low risk of bias & $\begin{array}{l}\text { Some } \\
\text { concerns }\end{array}$ \\
\hline 38) Zomer (2016) & Low risk of bias & Some concerns & Low risk of bias & Some concerns & Low risk of bias & $\begin{array}{l}\text { Some } \\
\text { concerns }\end{array}$ \\
\hline 39) Öncü (2019) & Some concerns & Some concerns & Low risk of bias & Low risk of bias & Some concerns & $\begin{array}{l}\text { Some } \\
\text { concerns }\end{array}$ \\
\hline
\end{tabular}

Note. NA = not applicable.

\section{Discussion}

This rapid review of interventions to increase personal protective behaviours to limit the spread of respiratory viruses identified 39 studies conducted across 15 countries. The majority of interventions targeted hand hygiene and/or face mask use, with one intervention targeting the catching of droplets in tissues in addition to hand hygiene. None of the identified interventions focused on avoiding touching the T-Zone, disinfecting surfaces or maintaining physical distancing. Interventions were typically delivered in participants' own homes or in nurseries/schools, targeting children/adult household members or pre- or school children/teachers. Two- or three-arm study designs with passive comparators were typically used. The overall quality of included studies was low, with only one study rated as 'low risk of bias'. The majority of interventions had a face-to-face component and delivered a median of three BCTs; the most frequent were 'Adding objects to the environment', 'Instruction on how to perform the behaviour' and 'Information about health consequences'. Where investigated, interventions were considered acceptable by participants, with a minority reporting issues with mask wear discomfort or skin irritation from hand hygiene products. Few studies reported the practicability, affordability, spill-over effects or equity of interventions. In a narrative synthesis, interventions targeting hand hygiene behaviour were found to have positive effects and those targeting face mask use had a mixture of positive and negative effects. Random-effects meta-analyses of a small number of studies found positive effects of interventions targeting hand hygiene behaviour and face mask use. However, between-study heterogeneity was high and the confidence interval for the pooled effect of interventions targeting face mask use was wide, partly due to the small number of studies included in the comparison. 


\section{Strengths and limitations}

This review was conducted rapidly (July-December 2020) with input on the research questions and review scope from public health and behavioural science experts and lay members as part of a written stakeholder consultation. However, the review has several limitations. First, given the expected large number of hand hygiene studies related to gastrointestinal infections, we limited the review to studies explicitly studying behaviour change in relation to respiratory viruses. However, data from interventions targeting personal protective behaviours to prevent gastrointestinal illness are likely to add to our understanding of the acceptability, effectiveness and equity of hand hygiene interventions. Second, data extraction was performed by a single reviewer, with a proportion verified by a second reviewer. Third, although most of identified studies were two- or three-arm RCTs, they were typically designed to study rates of respiratory infection as their primary outcome, with behaviour change outcomes less clearly reported. This hindered quantitative synthesis, with only a small number of included studies contributing to meta-analyses. Future studies specifically designed to examine the effectiveness of interventions on behavioural outcomes are needed. Fourth, in line with guidelines ${ }^{75}$, we only coded BCTs when there was clear evidence of their presence; interventions may have included additional BCTs not documented in this review.

\section{Implications for policy and practice}

Although we caution against drawing firm conclusions due to the low quality of the evidence, positive effects of interventions targeting hand hygiene behaviour and face mask use were observed, with the majority of interventions providing free hand hygiene products and/or face masks to participants in addition to instructions on how to perform the behaviour and information about health consequences. As far as is practicably feasible, authorities should aim to provide free products to staff, clients and visitors during respiratory viral epidemics. The limited range of BCTs included in interventions suggests a missed opportunity for harnessing techniques indicated by relevant behaviour change theory and evidence. We encourage policymakers and healthcare practitioners to work collaboratively with behavioural scientists to incorporate techniques that theory or evidence predicts are effective for enabling personal protective behaviours ${ }^{15}$, such as techniques targeting motivational or self-regulatory processes.

\section{Avenues for future research}

Findings highlight the need for evaluations of interventions to support people to avoid touching the T-Zone, disinfect surfaces, maintain physical distancing and ensure efficient ventilation. Ventilation is increasingly seen as an important personal protective behaviour but was missed from the present review as it was planned during an earlier epidemic phase when the emphasis was on viral transmission via droplets rather than aerosols ${ }^{76,77}$. In addition, we need studies designed to detect effects on behavioural outcomes and data on the affordability and equity of interventions to increase personal protective behaviours, particularly in low- and middle-income countries. Although the provision of hand hygiene products and face masks may offset costs related to primary and secondary care or work absenteeism for those with severe respiratory viral illness, the provision of free products at scale may be prohibitively costly. Future research involving health 
and social care economists should evaluate the cost-effectiveness of different types of interventions to enable personal protective behaviours, including those targeting motivational and self-regulatory processes. We also need further evidence from studies evaluating interventions to improve adherence to face mask use, with unclear results observed at present.

\section{Conclusions}

This rapid review identified 39 studies across 15 countries with interventions targeting hand hygiene and/or face mask use. Positive effects of interventions targeting hand hygiene were observed, with unclear results for interventions targeting face mask use.

\section{Funding}

OP receives salary support from Cancer Research UK (C1417/A22962).

\section{Declaration of interests}

OP, DS, EC, LS, RW and SM have no conflicts of interest to declare.

\section{Acknowledgements}

We gratefully acknowledge the funding listed. The funder had no final role in the study design; in the analysis and interpretation of data; in the writing of the report; or in the decision to submit the paper for publication. All researchers listed as authors are independent from the funder and all final decisions about the research were unrestricted.

\section{References}

1. West R, Michie S, Rubin J, Amlôt R. Don't touch the T-Zone-how to block a key pathway to infection with SARSCoV-2. BMJ Opinion. April 3, 2020.

2. Ferguson NM, Cummings DAT, Fraser C, Cajka JC, Cooley PC, Burke DS. Strategies for mitigating an influenza pandemic. Nature. 2006;442(7101):448-452. doi:10.1038/nature04795

3. Michie S, West R, Amlôt R. Behavioural strategies for reducing covid-19 transmission in the general population. BMJ Opinion. March 3, 2020.

4. Michie S, Rubin J, Amlôt R. Behavioural science must be at the heart of the public health response to covid-19. BMJ Opinion. February 28, 2020.

5. West R, Michie S, Rubin J, Amlôt R. How understanding principles of behaviour change can help limit the spread of COVID-19. submitted.

6. World Health Organisation. Non-Pharmaceutical Public Health Measures for Mitigating the Risk and Impact of 
Epidemic and Pandemic Influenza.; 2019.

7. Lunn P, Belton C, Lavin C, Mcgowan F, Timmons S, Robertson D. Using Behavioural Science to Help Fight the Coronavirus. Econ Soc Res Inst. 2020:1-24. https://www.esri.ie/publications/using-behavioural-science-to-help-fight-thecoronavirus.

8. Bish A, Michie S. Demographic and attitudinal determinants of protective behaviours during a pandemic: A review. Br J Health Psychol. 2010;15(4):797-824. doi:10.1348/135910710X485826

9. $\quad$ Michie S, Atkins L, West R. The Behaviour Change Wheel: A Guide to Designing Interventions. Silverback Publishing; 2014.

10. Michie S, West R, Amlôt R, Rubin J. Slowing down the covid-19 outbreak: changing behaviour by understanding it. BMJ Opinion. March 11, 2020.

11. International Monetary Fund. World Economic Outlook Update.; 2020.

https://www.imf.org/en/Publications/WEO/Issues/2020/06/24/WEOUpdateJune2020\#: :text=Global growth is projected at,more gradual than previously forecast.

12. Blake P, Wadhwa D. 2020 Year in Review: The impact of COVID-19 in 12 charts. World Bank Blog.

13. SafeLives. Domestic abuse and COVID-19.

14. Brooks SK, Webster RK, Smith LE, et al. The psychological impact of quarantine and how to reduce it: rapid review of the evidence. Lancet. 2020;395(10227):912-920. doi:10.1016/S0140-6736(20)30460-8

15. Warren-Gash C, Fragaszy E, Hayward AC. Hand hygiene to reduce community transmission of influenza and acute respiratory tract infection: a systematic review. Influenza Other Respi Viruses. 2012;7(5):738-749. doi:10.1111/irv.12015

16. Edwards R, Charani E, Sevdalis N, et al. Optimisation of infection prevention and control in acute health care by use of behaviour change: A systematic review. Lancet Infect Dis. 2012;12(4):318-329. doi:10.1016/S1473-3099(11)70283-3 17. Huis A, van Achterberg T, de Bruin M, Grol R, Schoonhoven L, Hulscher M. A systematic review of hand hygiene improvement strategies: a behavioural approach. Implement Sci. 2012;7(1):1-14. doi:10.1186/1748-5908-7-92

18. Luangasanatip N, Hongsuwan M, Limmathurotsakul D, et al. Comparative efficacy of interventions to promote hand hygiene in hospital: Systematic review and network meta-analysis. BMJ. 2015;351. doi:10.1136/bmj.h3728

19. Olena Doronina RN, Jones D, Martello M, Biron A, Lavoie-Tremblay M. A Systematic Review on the Effectiveness of Interventions to Improve Hand Hygiene Compliance of Nurses in the Hospital Setting. J Nurs Scholarsh.

2017;49(2):143-152. doi:10.1111/jnu.12274

20. Mbakaya BC, Lee PH, Lee RLT. Hand hygiene intervention strategies to reduce diarrhoea and respiratory infections among schoolchildren in developing countries: A systematic review. Int J Environ Res Public Health. 2017;14(4):1-14. doi:10.3390/ijerph14040371

21. Kwok YLA, Gralton J, McLaws ML. Face touching: A frequent habit that has implications for hand hygiene. Am J Infect Control. 2015;43(2):112-114. doi:10.1016/j.ajic.2014.10.015

22. World Health Organisation. Rapid Reviews to Strengthen Health Policy and Systems: A Practical Guide. (Tricco AC, Langlois E V, Straus SE, eds.); 2017. doi:10.1111/j.1365-2966.2008.12859.x

23. Tricco AC, Antony J, Zarin W, et al. A scoping review of rapid review methods. BMC Med. 2015;13:224. doi:10.1186/s12916-015-0465-6 
24. Haby MM, Chapman E, Clark R, Barreto J, Reveiz L, Lavis JN. What are the best methodologies for rapid reviews of the research evidence for evidence-informed decision making in health policy and practice: A rapid review. Heal Res Policy Syst. 2016;14(1):1-12. doi:10.1186/s12961-016-0155-7

25. West R, Michie S. Routes of transmission of SARS-CoV-2 and behaviours to block it: a summary. Qeios. 2020:1-4. https://www.qeios.com/read/article/563.

26. Norris E, Finnerty AN, Hastings J, Stokes G, Michie S. A scoping review of ontologies related to human behaviour change. Nat Hum Behav. 2019;3(2):164-172. doi:10.1038/s41562-018-0511-4

27. Michie S, Richardson M, Johnston M, et al. The behavior change technique taxonomy (v1) of 93 hierarchically clustered techniques: Building an international consensus for the reporting of behavior change interventions. Ann Behav Med. 2013;46(1):81-95. doi:10.1007/s12160-013-9486-6

28. Moore GF, Evans RE. What theory, for whom and in which context? Reflections on the application of theory in the development and evaluation of complex population health interventions. SSM - Popul Heal. 2017;3:132-135. doi:10.1016/j.ssmph.2016.12.005

29. Carey RN, Connell LE, Johnston M, et al. Behavior Change Techniques and Their Mechanisms of Action: A Synthesis of Links Described in Published Intervention Literature. Ann Behav Med. 2019;53:693-707. doi:10.1093/abm/kay078

30. The Cochrane Collaboration. Cochrane Handbook for Systematic Reviews of Interventions Version 5.1.0. [Updated March 2011]. (Higgins J, Green S, eds.).; 2011. www.cochrane-handbook.org. Accessed November 15, 2015.

31. Viechtbauer W. Conducting Meta-Analyses in R with the metafor Package. J Stat Softw. 2010;36(3):1-48.

32. Cohen J. Statistical Power Analysis for the Behavioral Sciences. 2nd ed. Hillsdale, NJ; 1988.

33. Cheung MWL. A Guide to Conducting a Meta-Analysis with Non-Independent Effect Sizes. Neuropsychol Rev. 2019;29:387-396.

34. White C, Kolble R, Carlson R, Lipson N, Dolan M. The effect of hand hygiene on illness rate among students in university residence halls. Am J Infect Control. 2003;31(6):364-370. doi:10.1067/mic.2003.76

35. Updegraff JA, Emanuel AS, Gallagher KM, Steinman CT. Framing Flu Prevention - An Experimental Field Test of Signs Promoting Hand Hygiene During the 2009-2010 H1N1 Pandemic. Heal Psychol. 2011;30(3):295-299. doi:10.1037/a0023125

36. Arbogast JW, Moore-Schiltz L, Jarvis WR, Harpster-Hagen A, Hughes J, Parker A. Impact of a Comprehensive Workplace Hand Hygiene Program on Employer Health Care Insurance Claims and Costs, Absenteeism, and Employee Perceptions and Practices. J Occup Environ Med. 2016;58(6). doi:10.1097/JOM.0000000000000738

37. Aiello AE, Murray GF, Perez V, et al. Mask Use, Hand Hygiene, and Seasonal Influenza-Like IIIness among Young Adults: A Randomized Intervention Trial. J Infect Dis. 2010;201(4):491-498. doi:10.1086/650396

38. Aiello AE, Perez V, Coulborn RM, Davis BM, Uddin M, Monto AS. Facemasks, hand hygiene, and influenza among young adults: A randomized intervention trial. PLoS One. 2012;7(1). doi:10.1371/journal.pone.0029744

39. Stedman-Smith M, DuBois CLZ, Grey SF, et al. Outcomes of a Pilot Hand Hygiene Randomized Cluster Trial to Reduce Communicable Infections Among US Office-Based Employees. J Occup Environ Med. 2015;57(4):374-380. doi:10.1097/JOM.0000000000000421 
40. Stebbins S, Stark JH, Vukotich Jr CJ. Compliance With a Multilayered Nonpharmaceutical Intervention in an Urban Elementary School Setting. J Public Heal Manag Pract. 2010;16(4):316-324.

41. Sandora TJ, Taveras EM, Shih M-C, et al. A Randomized, Controlled Trial of a Multifaceted Intervention Including Alcohol-Based Hand Sanitizer and Hand-Hygiene Education to Reduce Illness Transmission in the Home. Pediatrics. 2005;116(3):587-594. doi:10.1542/peds.2005-0199

42. Mott PJ, Sisk BW, Arbogast JW, Ferrazzano-Yaussy C, Bondi CAM, Sheehan JJ. Alcohol-Based Instant Hand Sanitizer Use in Military Settings: A Prospective Cohort Study of Army Basic Trainees. Mil Med. 2007;172:1170-1176. 43. Larson EL, Ferng Y-H, Wong-McLoughlin J, Wang S, Haber M, Morse SS. Impact of Non-Pharmaceutical Interventions on URIs and Influenza in Crowded, Urban Households. Public Health Rep. 2010;125:178-191. 44. Larson EL, Ferng Y-H, McLoughlin JW, Wang S, Morse SS. Effect of Intensive Education on Knowledge, Attitudes, and Practices Regarding Upper Respiratory Infections Among Urban Latinos. Nurs Res. 2009;58(3):150-157.

45. Koep TH, Jenkins S, Hammerlund MEM, et al. Promotion of Influenza Prevention Beliefs and Behaviors through Primary School Science Education. J Community Med Heal Educ. 2016;6(3). doi:10.4172/2161-0711.1000444

46. Azman AS, Stark JH, Althouse BM, et al. Household transmission of influenza A and B in a school-based study of non-pharmaceutical interventions. Epidemics. 2013;5(4):181-186. doi:10.1016/j.epidem.2013.09.001

47. Liu X, Zhao Z, Hou W, et al. A multimodal intervention to improve hand hygiene compliance via social cognitive influences among kindergarten teachers in China. PLoS One. 2019;14(5):e0215824.

48. Cowling BJ, Chan K-H, Fang VJ, et al. Facemasks and Hand Hygiene to Prevent Influenza Transmission in Households: A Cluster Randomized Trial. Ann Intern Med. 2009;151:437-446.

49. Cowling BJ, Fung ROP, Cheng CKY, et al. Preliminary findings of a randomized trial of non-pharmaceutical interventions to prevent influenza transmission in households. PLoS One. 2008;3(5). doi:10.1371/journal.pone.0002101 50. Chan SSC, So WKW, Wong DCN, Lee ACK, Tiwari A. Improving older adults' knowledge and practice of preventive measures through a telephone health education during the SARS epidemic in Hong Kong: A pilot study. Int J Nurs Stud. 2007;44:1120-1127. doi:10.1016/j.jnurstu.2006.04.019

51. Or PP-L, Ching PT-Y, Chung JW-Y. Can Flu-Like Absenteeism in Kindergartens Be Reduced Through Hand Hygiene Training for Both Parents and Their Kindergarteners? J Prim Care Community Health. 2020;11:1-6. doi:10.1177/2150132719901209

52. Suess T, Remschmidt C, Schink S, et al. Facemasks and intensified hand hygiene in a German household trial during the 2009/2010 influenza A (H1N1) pandemic: adherence and tolerability in children and adults. Epidemiol Infect. 2011;139:1895-1901. doi:10.1017/S0950268810003006

53. Suess T, Remschmidt C, Schink SB, et al. The role of facemasks and hand hygiene in the prevention of influenza transmission in households: Results from a cluster randomised trial; Berlin, Germany, 2009-2011. BMC Infect Dis. 2012;12(26). doi:10.1186/1471-2334-12-26

54. Hübner N-O, Hübner C, Wodny M, Kampf G, Kramer A. Effectiveness of alcohol-based hand disinfectants in a public administration: Impact on health and work performance related to acute respiratory symptoms and diarrhoea. BMC Infect Dis. 2010;10(250).

55. Simmerman JM, Suntarattiwong P, Levy J, et al. Findings from a household randomized controlled trial of hand 
washing and face masks to reduce influenza transmission in Bangkok, Thailand. Influenza Other Respi Viruses. 2011;5:256-267. doi:10.1111/j.1750-2659.2011.00205.x

56. Kaewchana S, Simmerman M, Somrongthong R, Suntarattiwong P, Lertmaharit S, Chotipitayasunondh T. Effect of Intensive Hand Washing Education on Hand Washing Behaviors in Thai Households with an Influenza-Positive Child in Urban Thailand. Asia-Pacific J Public Heal. 2012;24(4):577-585. doi:10.1177/1010539510393728

57. Apisarnthanarak A, Apisarnthanarak P, Cheevakumjorn B, Mundy LM. Intervention with an Infection Control Bundle to Reduce Transmission of Influenza-Like IIInesses in a Thai Preschool. Infect Control Hosp Epidemiol. 2009;30(9):1-6. doi:10.1086/599773

58. Roberts L, Smith W, Jorm L, Patel M, Douglas RM, McGilchrist C. Effect of Infection Control Measures on the Frequency of Upper Respiratory Infection in Child Care: A Randomized, Controlled Trial. Pediatrics. 2000;105(4):738-742. 59. MacIntyre CR, Cauchemez S, Dwyer DE, et al. Face mask use and control of respiratory virus transmission in households. Emerg Infect Dis. 2009;15(2):233-241. doi:10.3201/eid1502.081167

60. Nandrup-Bus I. Mandatory handwashing in elementary schools reduces absenteeism due to infectious illness among pupils: A pilot intervention study. Am J Infect Control. 2009;37(10):820-826. doi:10.1016/j.ajic.2009.06.012 61. Bundgaard H, Bundgaard JS, Raaschou-Pedersen DET, et al. Effectiveness of Adding a Mask Recommendation to Other Public Health Measures to Prevent SARS-CoV-2 Infection in Danish Mask Wearers. Ann Intern Med. 2020.

62. Azor-Martinez E, Cobos-Carrascosa E, Seijas-Vasquez ML, et al. Hand Hygiene Program Decreases School Absenteeism Due to Upper Respiratory Infections. J Sch Health. 2016;86(12):873-881.

63. Azor-Martinez E, Yui-Hifume R, Munoz-Vico FJ, et al. Effectiveness of a Hand Hygiene Program at Child Care Centers: A Cluster Randomized Trial. Pediatrics. 2018;142(5):e20181245.

64. Yardley L, Miller S, Schlotz W, Little P. Evaluation of a Web-Based Intervention to Promote Hand Hygiene: Exploratory Randomized Controlled Trial. J Med Internet Res. 2011;13(4):e107. doi:10.2196/jmir.1963

65. Little P, Stuart B, Hobbs FDR, et al. An internet-delivered handwashing intervention to modify influenza-like illness and respiratory infection transmission (PRIMIT): a primary care randomised trial. Lancet. 2014;386(10004):1631-1639. doi:10.1016/S0140-6736(15)60127-1

66. Barasheed O, Almasri N, Badahdah A-M, et al. Pilot Randomised Controlled Trial to Test Effectiveness of Facemasks in Preventing Influenza-like Illness Transmission among Australian Hajj Pilgrims in 2011. Infect Disord - Drug Targets. 2014;14:110-116. doi:10.2174/1871526514666141021112855

67. Ram PK, Divita MA, Khatun-e-Jannat K, et al. Impact of Intensive Handwashing Promotion on Secondary Household Influenza-Like Illness in Rural Bangladesh: Findings from a Randomized Controlled Trial. PLoS One. 2015;10(6):e0125200. doi:10.1371/journal.pone.0125200

68. Reyes Fernández B, Lippke S, Knoll N, Moya EB, Schwarzer R. Promoting action control and coping planning to improve hand hygiene. BMC Public Health. 2015;15(964). doi:10.1186/s12889-015-2295-z

69. Savolainen-Kopra C, Haapakoski J, Peltola PA, et al. Hand washing with soap and water together with behavioural recommendations prevents infections in common work environment: an open cluster-randomized trial. Trials. 2012;13(10). 70. Canini L, Andréoletti L, Ferrari P, et al. Surgical mask to prevent influenza transmission in households: A cluster randomized trial. PLoS One. 2010;5(11):1-6. doi:10.1371/journal.pone.0013998 
71. Zomer TP, Erasmus V, Looman CW, et al. Improving hand hygiene compliance in child daycare centres: a randomized controlled trial. Epidemiol Infect. 2016;144:2552-2560. doi:10.1017/S0950268816000911

72. Öncü E, Köksoy Vayısoğlu S, Lafci D, Yurtsever D, Ravlı Bulut E, Peker E. Comparison of Interactive Education Versus Fluorescent Concretization on Hand Hygiene Compliance Among Primary School Students: A Randomized Controlled Trial. J Sch Nurs. 2019;35(5):337-347. doi:10.1177/1059840518785447

73. Cowling, B.J., Chan, K.H., FAng, V.J., Cheng CKY. Facemasks and Hand Hygiene to Prevent Influenza Transmission in Households. Ann Intern Med. 2009;(151):437-446.

74. Aiello AE, Murray GF, Perez V, et al. Mask Use, Hand Hygiene, and Seasonal Influenza-Like IIIness among Young Adults: A Randomized Intervention Trial. J Infect Dis. 2010;201:491-498. doi:10.1086/650396

75. Wood CE, Richardson M, Johnston M, et al. Applying the behaviour change technique (BCT) taxonomy v1: a study of coder training. Trans/ Behav Med. 2015;5:134-148. doi:10.1007/s13142-014-0290-z

76. Anderson EL, Turnham P, Griffin JR, Clarke CC. Consideration of the Aerosol Transmission for COVID-19 and Public Health. Risk Anal. 2020;40(5):902-907. doi:10.1111/risa.13500

77. Morawska L, Milton DK. It Is Time to Address Airborne Transmission of Coronavirus Disease 2019 (COVID-19). Clin Infect Dis. 2020;71:2311-2313. doi:10.1093/cid/ciaa939 